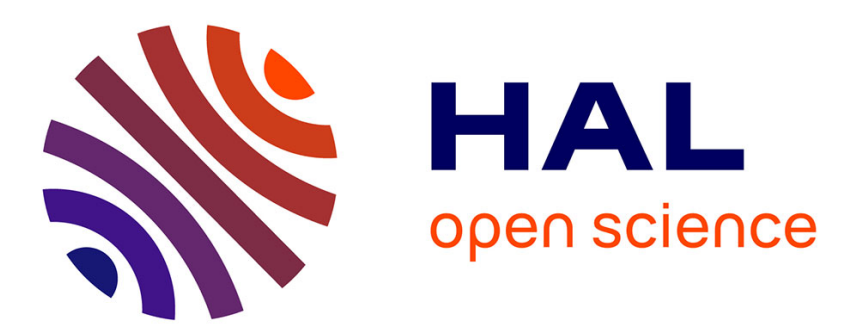

\title{
Multiscale magneto-elastic modeling of magnetic materials including isotropic second order stress effect \\ Olivier Hubert
}

\section{To cite this version:}

Olivier Hubert. Multiscale magneto-elastic modeling of magnetic materials including isotropic second order stress effect. Journal of Magnetism and Magnetic Materials, 2019, 491, pp.165564. 10.1016/j.jmmm.2019.165564 . hal-02196167

\section{HAL Id: hal-02196167 \\ https://hal.science/hal-02196167}

Submitted on 26 Jul 2019

HAL is a multi-disciplinary open access archive for the deposit and dissemination of scientific research documents, whether they are published or not. The documents may come from teaching and research institutions in France or abroad, or from public or private research centers.
L'archive ouverte pluridisciplinaire HAL, est destinée au dépôt et à la diffusion de documents scientifiques de niveau recherche, publiés ou non, émanant des établissements d'enseignement et de recherche français ou étrangers, des laboratoires publics ou privés. 


\title{
Multiscale magneto-elastic modeling of magnetic materials including isotropic second order stress effect
}

\author{
Olivier HUBERT ${ }^{\mathrm{a}, *}$ \\ ${ }^{a}$ LMT (ENS Paris-Saclay/CNRS/Université Paris-Saclay) \\ 61, avenue du président Wilson, 94235 Cachan cedex, France
}

\begin{abstract}
Compact and high speed electromechanical systems lead to higher and higher levels of multiaxial mechanical stress, that may strongly change the magnetic behavior of materials, making the development of highly accurate magnetic models a very important task. Among all available magnetoelastic models, multiscale approaches seem to be the most promising. The coupling effect is introduced at the single crystal scale, mainly attributed to the evolution of magnetic domain structures under magneto-mechanical loading. All these models use however a formulation of free energy where the energetic term describing magneto-elastic coupling is linearly stress dependent, that hardly allow (using artificial mechanisms) providing non-monotonic stress effect on the magnetic behavior. The proposition detailed in this paper is to consider a second order stress term in the free energy expression, allowing a linear dependance of magnetostriction tensor with stress to be defined and providing the non-monotonous stress effect. This introduction leads to a more complex description of magnetoelastic effect that needs the identification of a large number of complementary material constants. In this paper, developments are made in the frame of cubic symmetry for first order magneto-elastic term (joining the classical description) and considering an isotropic second order stress effect for the sick of simplicity. This simplification leads to only two additive physical constants to be identified. An identification procedure is proposed and applied to model the magnetoelastic behavior a non-oriented (NO) 3wt\%silicon-iron electrical steel.
\end{abstract}

Keywords: magneto-elasticity, morphic effect, magnetostriction, multiscale modeling, iron-silicon alloys

\section{Introduction}

The search for weight optimization of electromechanical systems leads to build more compact and high speed systems (see [1] and reviews [2, 3, 4]).

\footnotetext{
*Corresponding author : O. Hubert, tel : ++ 33147402224 ; e-mail : hubert@lmt.enscachan.fr
}

Preprint submitted to Elsevier

July 16, 2019 
These systems generate high levels of multiaxial mechanical stress [5], that strongly change the magnetic behavior of materials as uniaxial stress can do $[6,7]$. The prediction of multiaxial stress effect encountered requires the use of multiaxial model where an accurate relation between stress and magnetic quantities (magnetic permeability and losses) is looked for. Few models are available. Macroscopic phenomenological approaches (see for instance [8, 9, 10, 11, 12, 13]) do not provide a sufficient description of such complex phenomena. Equivalent stress approaches where a full stress tensor is represented by a scalar value are interesting but usually use some restrictive hypotheses (isotropy, plane stress) $[14,15,16]$. Multiscale approaches where the coupling effect is introduced at the single crystal scale constitute an promising alternative way $[17,18,19,20,21,22,23]$ despite longer calculation times (that remains much lower than for micromagnetics). They seem relevant to establish macroscopic constitutive laws, able to be introduced in finite element calculations [24], since magneto-elastic coupling effects are mainly attributed to the evolution of magnetic domain structures under magneto-mechanical loading. Some of these models have been extended to predict hysteresis [21, 23].

Experiences show on the other hand that mechanical stress sometimes has a non-monotonic effect on magnetic behavior [10, 23, 25], leading to an increase followed by a decrease of magnetization at increasing stress level. This concerns, for example, iron-silicon alloys constitutive of most of high and medium-range electrical machines and sometimes subjected to high operating stresses [5]. The non-monotonic effect may concern different iron-based ferromagnetic materials when they are subjected to plastic deformation and suffer the effect of high intensity multiaxial internal stresses [26]. Unlike the well-known Villari reversal, it may appear at the lowest magnetic field levels (e.g. sudden decrease of initial susceptibility with increasing stress). To address this non-monotony, a concept of demagnetizing-stress field has been introduced by several authors (see [10, 23], and more recently in [25] for macroscopic attempts). Introduction in a multiscale model has been proposed but the variations of magnetization curves are usually not in good accordance with experimental results on the one hand. This theory does not explain the associated apparent change of magnetostriction sign with stress on the other hand $[23,27]$. To the best knowledge of author, micromagnetic models including magneto-elastic effects do not allow this phenomenon to be depicted either.

Indeed, all these models, from micro to macroscale, use a formulation of free energy where the energetic term describing magneto-elastic coupling is linearly stress dependent (using a uniaxial, plane stress or multiaxial stress description), in an isotropic or cubic frame. The proposition detailed in this paper is to consider a second order stress term in the free energy expression able to address the non-monotonic effect. Second order stress has initially been discussed in $[28,29]$ as a so-called morphic effect. This term allows a linear dependence of magnetostriction tensor with stress to be defined. The stiffness tensor becomes magnetic dependent as a secondary consequence leading to a part (usually neglected) of the so-called $\Delta E$ effect $[30,31]$. By introducing a new term in the Helmholtz free energy, a new expression of magnetoelastic energy is obtained 
and can be introduced in a multiscale modeling. This introduction leads to a more complete (but more complex) description of magnetoelastic effects in iron based material allowing to describe the non-monotonic change of permeability with stress and the change of magnetostriction sign.

In this paper, developments are made in the frame of cubic symmetry for first order magneto-elastic term (joining the classical description) and considering an isotropic second order stress effect. This simplification leads to a reduced number of additive physical constants to be identified. An identification procedure is proposed using a semi-analytical modeling of the magneto-elastic behavior. Finally, only two new constants are introduced allowing to extend the applicability of multiscale magneto-mechanical modeling of this class of materials to a wider stress range. The complete procedure is applied to a non-oriented (NO) 3wt.\%silicon-iron electrical steel.

The first part of the paper presents an experimental work where the nonmonotonic variation of magnetic behavior with stress is illustrated. The multiscale modeling and associated thermodynamic background are presented in the second part, where the second-order stress term is defined and introduced in the Gibbs free energy expression. The last part details the identification procedure of second order terms reduced to two parameters and illustrates some comparisons between modeling and experiments, showing the relevancy of this new approach.

\section{Influence of stress on magnetic and magnetostrictive behavior of a classical iron-silicon electrical steel}

\subsection{Experimental procedure and material}

The benchmark for magneto-mechanical measurements under uniaxial stress is based on a non-standard experimental frame. It is constituted of two face-toface positioned ferrimagnetic U-yokes (figure 1). Samples usually consist of 200 to $250 \mathrm{~mm}$ long and about $12.5 \mathrm{~mm}$ wide strips whose thickness can vary from $0.2 \mathrm{~mm}$ to few millimeters. Samples are instrumented with longitudinal and transverse strain gauges stuck on both sides in order to perform magnetostriction strain measurement (measurements got on both sides are averaged). A half Wheatstone bridge configuration with temperature compensation and low-pass second order butterworth filtering allows the strain gauges signals to be appropriately stored [32]. Digitizing is ensured by a synchronized NI-DAQ 16bit card. A primary winding is placed on the specimen. We restrict experiments to reversible behavior with usual methods: anhysteretic magnetization and magnetostriction curves are built point by point by applying a sinusoidal magnetic field (sinusoidal current in practice) superimposed to a bias field defining the anhysteretic point, and of exponentially decreasing amplitude. The measurement system is current controlled by a Kepco supply driven by a NI controller and home-made Labview software [33]. B-coil and H-coil ensure the measurement of magnetic parameters. The set-up is especially designed to get magnetomechanical reference results at very constant stress. The loading system is 
ensured by heavy weights connected to the sample by cables and articulated jaws stuck to the sample. This technique strongly reduces vibrations usually encountered in electro-hydraulic machines. The maximal load is $50 \mathrm{Kg}$ leading to a maximal stress of about $100 \mathrm{MPa}$ (depending on the section).

A commercial NO 3 wt.\%silicon-iron (0.5mm thick) lamination from ArcelorMittal was used for the experimental campaign. It is well known that NO FeSi exhibits a crystallographic texture $(\{111\}<\overline{2} 11>$ type crystallographic texture along the rolling direction (RD)) leading to an orthotropic magnetic and magnetostrictive behavior already discussed in [32, 34, 35]. Anisotropic phenomena, even reachable by experiments and modeling will not be discussed herein. Results reported in this paper concern strips cut by electro erosion in the lamination along the transverse direction (TD) where the most significant magnetostriction strains amplitude are obtained.

\subsection{Experimental results}

Figure 2 shows the influence of the magnetic loading $H$ on the magnetization $M$ at different stress levels $\sigma$ between 0 and $78 \mathrm{MPa}$ (some interesting stress levels are highlighted, others are not labeled). Two representations are proposed: figure 2a illustrates the magnetization curves obtained at constant stress; figure $2 \mathrm{~b}$ shows the evolution of the magnetization as a function of stress for different magnetic field amplitudes. These figures are complemented by figure 3a which shows the evolution of the derivative of the magnetization with respect to the stress, and by figure 3b which shows the evolution of the initial susceptibility (anhysteretic) with stress. This set of results illustrates well the purpose of the paper: below 15 to $20 \mathrm{MPa}$, a mechanical stress leads to an increase in the magnetization from the lowest magnetic fields up to about $2000 \mathrm{~A} / \mathrm{m}$. Higher magnetic field levels cause the well-known mechanism of magnetization rotation and associated reverse sensitivity of magnetization to stress. This is the Villari effect resulting in a crossing of the high-field magnetization curves (illustrated in figure $2 \mathrm{~b}$ for $H=10000 \mathrm{~A} / \mathrm{m}$ ). This Villari effect (so-called Villari reversal) is well documented especially for pure iron and various steels $[6,7,8,36,37]$. Its modeling is generally possible when crystalline anisotropy and magnetization rotation are taken into account $[38,39,37,26]$. For stresses greater than $20 \mathrm{MPa}$, the sensitivity of the magnetization to stress is negative whatever the magnetic field level, including zero magnetic field as shown in figure $3 \mathrm{~b}$. These results are perfectly in agreement with the experimental results reported in some recent communications [12, 25, 27].

The figure 4 shows the concomitant evolution of the longitudinal magnetostriction deformation $(/ / \vec{H}$ - figure $4 \mathrm{a})$ and transverval magnetostriction deformation $(\perp \vec{H}$ - figure $4 \mathrm{~b})$ as a function of the magnetization. These results are consistent with former results available in literature. Several concordances can be observed with the magnetic behavior. Thus, for a stress less than 15-20 $\mathrm{MPa}$, the longitudinal deformation increases with magnetization up to levels corresponding to the Villari reversal (in agreement with the crossing of the high-field magnetization curves). At higher stress, the sample shrinks in the 
direction of the applied magnetic field. This contraction increases as the stress increases but tends to reach a kind of saturation level. It is observed, however, that at high magnetization the rotation mechanism and the associated variation in deformation seem preserved, which would mean that this mechanism is decorrelated from the mechanical stresses. In general, the transverse deformation is of opposite sign to the longitudinal deformation. Its variations are also always opposite to those observed in the longitudinal direction. The lack of information across the thickness does not allow us to conclude with regard to the volume conservation during the magnetization, even if this assumption is usually retained $[6,7,40]$. 
These measurements perfectly illustrate the non-monotonic effect of the stresses on the magnetic behavior. This behavior is clearly correlated with the observed magnetostriction variations. Numerical simulations have confirmed that magnetic forces can not be incriminated. This result is indirectly confirmed by similar magnetic and magnetostrictive measurements performed on an ironcobalt alloy and obtained using the same set-up, where no non-monotonic effect has been observed $[41,16]$. The correlation between magnetostriction variation and magnetization variation seems, on the contrary, to indicate that the mechanism can only be modeled through a suitable constitutive law. In this case, the use of a demagnetizing stress field can only bring about a partial correction of the magnetization levels but can not in any case produce a significant effect on the magnetostriction behavior on the one hand and be extended to a multiaxial mechanical loading on the other hand. Introduction of a higher order stress term in the constitutive law is key.

\section{Multiscale modeling}

A multiscale model of a representative volume element (RVE) of such materials is presented in this section including the second order stress effect, remaining in the framework of continuum mechanics. In the following, $g$ denotes a grain. It is supposed to be composed of several phases $\phi$ (typically ferrite, austenite, martensite,...), that may be separated in different variants (see [42, 43] for more details) but remain at constant volume fraction in the modeling proposed ${ }^{1} . \alpha$ denotes a magnetic domain family inside the ferromagnetic phase considered and represents the lower scale. The scale organization is illustrated in figure 5, from the RVE to the domain scale, and corresponds to scales where some physical fields can be considered as homogeneous leading to some simplifications in the energy description (recalling the assumptions of former phase model from Néel [45]). An implicit method is employed to homogenize the magnetic, mechanical and magnetostrictive behavior $[22,46]$. A first step consists to build an energy function at the domain scale where anisotropic crystallographic phenomena are significant and some fields can be simplified. Indeed the selection of a domain family by energy minimization complemented by the magnetization rotation are considered as the two phenomenons that precondition the macroscopic behavior.

\subsection{Governing equations and Gibbs free energy at the domain scale}

The first and second law of thermodynamics are considered in the frame of small mechanical perturbations and in the absence of internal heat generation (e.g. Eddy currents are not considered). The first principle given at the local scale $\left(\mathrm{J} / \mathrm{m}^{3}\right)$ express that the total energy density composed of kinetic energy density and internal energy density can only change by the action of external

\footnotetext{
${ }^{1}$ the modeling applies to single or multiphased materials where one phase at minimum is ferro(ferri)magnetic but remains at constant volume fraction. Extension to phase change is possible and applies for magnetic shape memory alloys for example [44].
} 
work and heat exchange. $u_{\alpha}$ denotes the internal energy density at the domain scale. At constant velocity, kinetic energy is constant. There is a direct relationship between the variation of internal energy density and power sources following:

$$
\dot{u}_{\alpha}=\sigma_{\alpha}: \dot{\boldsymbol{\epsilon}}_{\alpha}+\mu_{0} \dot{\vec{M}}_{\alpha} \cdot \vec{H}_{\alpha}-\operatorname{div}\left(\vec{q}_{\alpha}\right)
$$

$\sigma_{\alpha}$ and $\dot{\epsilon}_{\alpha}$ are the local stress and strain rate tensors (bold format refers to second order tensor) whose double contraction forms the mechanical power density. $\vec{M}_{\alpha}$ and $\vec{H}_{\alpha}$ refer to the local magnetization and magnetic field vectors (the superscript arrow refers to first order tensor $=$ vector). The magnetization rate times magnetic field multiplied by the vacuum permeability $\mu_{0}$ define the magnetic power ${ }^{2} \cdot \vec{q}_{\alpha}$ is the heat flux vector.

The second law of thermodynamics states that:

$$
T_{\alpha} \dot{s}_{\alpha} \geq-\operatorname{div}\left(\vec{q}_{\alpha}\right)+\frac{1}{T_{\alpha}} \vec{q}_{\alpha} \cdot \overrightarrow{\operatorname{grad}} T_{\alpha}
$$

with $T_{\alpha}$ the local temperature and $s_{\alpha}$ the entropy density. Introducing the Helmholtz free energy density,

$$
\psi_{\alpha}=u_{\alpha}-T_{\alpha} s_{\alpha}
$$

the so-called Clausius-Duhem inequality is obtained and decomposed in intrinsic (4) and extrinsic (5) parts following:

$$
\begin{gathered}
\boldsymbol{\sigma}_{\alpha}: \dot{\boldsymbol{\epsilon}}_{\alpha}+\mu_{0} \dot{\vec{M}}_{\alpha} \cdot \vec{H}_{\alpha}-\left(s_{\alpha} \dot{T}_{\alpha}+\dot{\psi}_{\alpha}\right) \geq 0 \\
-\frac{1}{T_{\alpha}} \vec{q}_{\alpha} \cdot \overrightarrow{\operatorname{grad}} T_{\alpha} \geq 0
\end{gathered}
$$

The second expression is unused since thermal effects are not considered (isothermal problem). The Helmholtz free energy is a function of deformation, magnetization and temperature as control variables $\psi_{\alpha}=\psi_{\alpha}\left(\boldsymbol{\epsilon}_{\alpha}, \vec{M}_{\alpha}, T_{\alpha}\right)$. The associated power is consequently:

$$
\dot{\psi}_{\alpha}=\frac{\partial \psi_{\alpha}}{\partial \boldsymbol{\epsilon}_{\alpha}}: \dot{\boldsymbol{\epsilon}}_{\alpha}+\frac{\partial \psi_{\alpha}}{\partial \vec{M}_{\alpha}} \cdot \dot{\vec{M}}_{\alpha}+\frac{\partial \psi_{\alpha}}{\partial T_{\alpha}} \dot{T}_{\alpha}
$$

The reversible framework states that the first intrinsic part of ClausiusDuhem inequality is null, leading to the so-called thermodynamic consistency equations (7).

\footnotetext{
${ }^{2}$ The magnetic power is usually defined as a scalar product of magnetic induction rate with magnetic field: $\dot{\vec{B}}_{\alpha} \cdot \vec{H}_{\alpha}$. It can be simplified using the decomposition $\vec{B}_{\alpha}=\mu_{0}\left(\vec{H}_{\alpha}+\vec{M}_{\alpha}\right)$ and considering that the contribution of the magnetic field to the total power is negligible because it is present even in the absence of magnetization (see [47, 48]).
} 


$$
\boldsymbol{\sigma}_{\alpha}=\frac{\partial \psi_{\alpha}}{\partial \boldsymbol{\epsilon}_{\alpha}} \quad ; \quad \vec{H}_{\alpha}=\frac{\partial \psi_{\alpha}}{\mu_{0} \partial \vec{M}_{\alpha}} \quad ; \quad s_{\alpha}=-\frac{\partial \psi_{\alpha}}{\partial T_{\alpha}}
$$

Therefore, the Helmholtz free energy variation (for a time step $d t$ ) is controlled by magnetization and total deformation variations at constant temperature:

$$
d \psi_{\alpha}=\boldsymbol{\sigma}_{\alpha}: d \boldsymbol{\epsilon}_{\alpha}+\mu_{0} \vec{H}_{\alpha} \cdot d \vec{M}_{\alpha}
$$

The magnetic free enthalpy (Legendre transformation of magnetic quantity) $\kappa_{\alpha}=\psi_{\alpha}-\mu_{0} \vec{H}_{\alpha} \cdot \vec{M}_{\alpha}$ allows the energy variation to be defined as function of magnetic field variation instead of induction variation. The Gibbs free energy (or free enthalpy) (Legendre transformation of mechanical quantity) $g_{\alpha}=\kappa_{\alpha}-\boldsymbol{\sigma}_{\alpha}$ : $\boldsymbol{\epsilon}_{\alpha}$ allows finally the energy variation to be defined as function of stress variation instead of deformation variation. Variation of Gibbs free energy is given by:

$$
d g_{\alpha}=-\boldsymbol{\epsilon}_{\alpha}: d \boldsymbol{\sigma}_{\alpha}-\mu_{0} \vec{M}_{\alpha} \cdot d \vec{H}_{\alpha}
$$

Strain and magnetic induction finally derive from the Gibbs free energy function following:

$$
\boldsymbol{\epsilon}_{\alpha}=-\frac{\partial g_{\alpha}}{d \boldsymbol{\sigma}_{\alpha}} \quad ; \quad \vec{M}_{\alpha}=-\frac{\partial g_{\alpha}}{\mu_{0} \partial \vec{H}_{\alpha}}
$$

Small perturbation hypothesis allows the total deformation to be considered as a sum of elastic $\epsilon_{\alpha}^{e}$ and magnetostriction $\epsilon_{\alpha}^{\mu}$ strains leading to:

$$
\boldsymbol{\epsilon}_{\alpha}=\boldsymbol{\epsilon}_{\alpha}^{e}+\boldsymbol{\epsilon}_{\alpha}^{\mu}=\mathbb{C}_{\alpha}^{-1}: \boldsymbol{\sigma}_{\alpha}+\boldsymbol{\epsilon}_{\alpha}^{\mu}
$$

$\mathbb{C}_{\alpha}$ is the fourth order local stiffness tensor. $\epsilon_{\alpha}^{\mu}$ may depend at this step from magnetic field and stress at the local scale so that the derivation of the mechanical part of the Gibbs free energy uses and integration step of the magnetostriction strain over the stress path:

$$
g_{\alpha}^{\text {mech }}=-\frac{1}{2} \boldsymbol{\sigma}_{\alpha}: \mathbb{C}_{\alpha}^{-1}: \boldsymbol{\sigma}_{\alpha}-\int_{\mathbf{0}}^{\boldsymbol{\sigma}_{\alpha}} \boldsymbol{\epsilon}_{\alpha}^{\mu}: d \boldsymbol{\sigma}_{\alpha}
$$

For the derivation of the magnetic part of the Gibbs free energy, the Helmholtz magnetic free energy $\psi_{\alpha}^{\text {magn }}$ is first defined as a Taylor expansion of magnetization (sixth order expansion), taking into account the fact that the magnetic behavior is an odd function of magnetization. Second-rank $\mathbf{K}_{\alpha}$, fourth-rank $\mathbb{K}_{\alpha}$ and sixth-rank $\mathcal{K}_{\alpha}$ tensors are used to define the magneto-crystalline part of Helmholtz free energy. Their expressions are strongly correlated to material symmetries $[28,49]$. The coupling between neighboring atoms leads to a constant magnetization direction over a wide volume (i.e. magnetic domains). Any angular deviation (especially inside domain walls) leads to an energy increase, known as the exchange energy, whose expression involves the magnetization gradient $\nabla \vec{M}_{\alpha}$ and an exchange constant $A$ in the framework of continuum 
mechanics [50]. The expression of Gibbs magnetic free energy (after Legendre transformation) is finally given by:

$g_{\alpha}^{m a g}=\frac{A}{M_{\alpha}^{2}} \nabla \vec{M}_{\alpha}: \nabla \vec{M}_{\alpha}+\vec{M}_{\alpha} \cdot \mathbf{K}_{\alpha} \cdot \vec{M}_{\alpha}+\vec{M}_{\alpha} \otimes \vec{M}_{\alpha}: \mathbb{K}_{\alpha}: \vec{M}_{\alpha} \otimes \vec{M}_{\alpha}+\vec{M}_{\alpha} \cdot\left(\vec{M}_{\alpha} \otimes \vec{M}_{\alpha}: \mathcal{K}_{\alpha}: \vec{M}_{\alpha} \otimes \vec{M}_{\alpha}\right) \cdot \vec{M}_{\alpha}-\mu_{0} \vec{H}_{\alpha}$

The sum of $g_{\alpha}^{\text {mech }}$ and $g_{\alpha}^{\text {mag }}$ gives the total Gibbs free energy of medium that must be minimum with respect to stress and magnetic field at the equilibrium. It must be noticed that the magneto-mechanical part of the mechanical Gibbs free energy could be defined in the frame of magnetic Gibbs free energy expression using a Taylor expansion as well [28]. Indeed this term denotes the coupling between magnetics and mechanics. Its expression is key for an appropriate modeling of the experimental observations made in the previous section.

\subsection{Simplification of Gibbs free energy at the domain scale}

Following the proposed scaling, $\alpha$ denotes now a domain family (or more simply a domain), whose number is defined by the number of easy magnetic axes in the crystal $(6$ directions for $<100>$ easy axes materials, and 8 directions for $<111>$ easy axes materials). It is first interesting to observe that the magnetization at the domain scale oriented along an axis $\vec{\gamma}_{\alpha}$ has a constant norm equal to the saturation magnetization $M_{s}$ [50]. The magnetization vector can be defined using the direction cosines $\gamma_{i}$ in the local frame (usually crystal frame $\vec{e}_{i}$ ) as:

$$
\vec{M}_{\alpha}=M_{s} \vec{\gamma}_{\alpha}=M_{s}{ }^{t}\left[\gamma_{1} \gamma_{2} \gamma_{3}\right]=M_{s} \gamma_{i} \vec{e}_{i}
$$

In a magnetic domain, spatial variations of magnetization are inexistent, the magnetization gradient is consequently zero. The Gibbs free energy (average energy density over a domain) can consequently be simplified but will not allow the domain walls to be described ${ }^{3}$.

The dyadic product of $\vec{M}_{\alpha}$ allows the second-rank orientation tensor $\boldsymbol{R}_{\alpha}$ to de defined :

$$
\vec{M}_{\alpha} \otimes \vec{M}_{\alpha}=M_{s}^{2} \vec{\gamma}_{\alpha} \otimes \vec{\gamma}_{\alpha}=M_{s}^{2}\left(\begin{array}{ccc}
\gamma_{1}^{2} & \gamma_{1} \gamma_{2} & \gamma_{1} \gamma_{3} \\
\gamma_{1} \gamma_{2} & \gamma_{2}^{2} & \gamma_{2} \gamma_{3} \\
\gamma_{1} \gamma_{3} & \gamma_{2} \gamma_{3} & \gamma_{3}^{2}
\end{array}\right)=M_{s}^{2} \boldsymbol{R}_{\alpha}
$$

Using the cubic symmetry and the identity $\sum_{i} \gamma_{i} \gamma_{i}=1$, the magnetic part of Gibbs free energy simplifies into [28, 50]:

$$
g_{\alpha}^{m a g}=K_{0}+K_{1}\left(\gamma_{1}^{2} \gamma_{2}^{2}+\gamma_{1}^{2} \gamma_{3}^{2}+\gamma_{2}^{2} \gamma_{2}^{2}\right)+K_{2}\left(\gamma_{1}^{2} \gamma_{2}^{2} \gamma_{3}^{2}\right)-\mu_{0} \vec{H}_{\alpha} \cdot \vec{M}_{\alpha}
$$

\footnotetext{
${ }^{3}$ This is addressed by micromagnetic formulations $[49,50]$
} 
where $K_{0}, K_{1}$ and $K_{2}$ are the so-called magnetocrystalline constants (defining the magnetocrystalline -non convex- energy density) and the last term is usually called Zeemann energy density (if $\vec{H}_{\alpha}$ is considered homogeneous [50, 22]).

The mechanical part of the Gibbs free energy may be simplified at the domain scale by using the so-called magnetostriction tensor of a domain $\alpha$ defined by [40]:

$$
\boldsymbol{\epsilon}_{\alpha}^{\mu}=\frac{3}{2}\left(\begin{array}{ccc}
\lambda_{100}\left(\gamma_{1}^{2}-\frac{1}{3}\right) & \lambda_{111} \gamma_{1} \gamma_{2} & \lambda_{111} \gamma_{1} \gamma_{3} \\
\lambda_{111} \gamma_{1} \gamma_{2} & \lambda_{100}\left(\gamma_{2}^{2}-\frac{1}{3}\right) & \lambda_{111} \gamma_{2} \gamma_{3} \\
\lambda_{111} \gamma_{1} \gamma_{3} & \lambda_{111} \gamma_{2} \gamma_{3} & \lambda_{100}\left(\gamma_{3}^{2}-\frac{1}{3}\right)
\end{array}\right)
$$

where $\lambda_{100}$ and $\lambda_{111}$ are the magnetostriction constants that correspond to the deformation of a perfect single crystal along $<100>$ and $<111>$ axes respectively at the magnetic saturation. This tensor is considered independent to stress and leads to the following reformulation of the mechanical part of the Gibbs free energy by allowing the integral term to be simplified ${ }^{4}$ :

$$
g_{\alpha}^{m e c h}=-\frac{1}{2} \boldsymbol{\sigma}_{\alpha}: \mathbb{C}_{\alpha}^{-1}: \boldsymbol{\sigma}_{\alpha}-\boldsymbol{\sigma}_{\alpha}: \boldsymbol{\epsilon}_{\alpha}^{\mu}
$$

To the best knowledge of author, multiscale or macroscopic models that use a linear stress dependent magneto-elastic energy are not able to depict the nonmonotonic effect, as observed experimentally. Micromagnetic models are no exception despite a more realistic description of the microstructure by taking into account demagnetizing fields. Some authors tried to propose a correction by implementing a stress dependent demagnetizing field [23, 27]. This solution is not satisfactory because it corresponds to the addition of an artificial weak coupling.

\subsection{Implementation of a second order stress term in the Gibbs free energy}

We have first to recall that magnetostriction at the local scale (leading typically to $\lambda_{100}$ and $\lambda_{111}$ parameters) is explained by the so-called spin-orbit coupling $[49,40]$. Spin orbit coupling means that the spin moment of an electron and its orbital moment are coupled. Indeed the electron's orbit around the atomic nucleus creates a magnetic field that modifies the direction of its spin magnetic moment (the magnetic moment wants to align on the magnetic field direction). Therefore, any change of magnetic field (effective field) leads to a reorientation of the electron's spin. But the orbital motion of the electron is also coupled with the lattice sites, that is called the orbital-lattice coupling. The main consequence is that if the orbital motion around the atomic nucleus is changed, the atomes position moves through the strong orbit-lattice coupling and leads to a deformation. It is called magnetostriction strain. Considering an initial undeformed state, magnetostriction depends on the orientation

\footnotetext{
${ }^{4}$ This simplification is especially encountered in the construction of macroscopic models $[9,51]$ but not always justified.
} 
of saturation magnetization. Indeed, spins reorient in a given direction, and the resultant orbital-lattice coupling leads to deformation that may be different from one direction to another (and may be from negative to positive value: e.g. iron $\left.-\lambda_{100}=21 \times 10^{-6}, \lambda_{111}=-21 \times 10^{-6}[40]\right)$. Because stress at the crystallographic scale leads to a distorsion of lattice, the magnetostriction that can be reached may be different at a given stress level and leads to significant changes of magnitude. It can be recalled at this step that the theoretical magnetostriction of an isotropic body is expressed as [31]:

$$
\lambda_{\text {sat }}=\frac{2}{5} \lambda_{100} k^{a}+\frac{3}{5} \lambda_{111} k^{b}
$$

$k^{a}$ and $k^{b}$ are homogenization parameters depending on the elastic properties. They are given by:

$$
k^{a}=\frac{\mu_{a}}{\mu_{e f f}} \frac{\mu_{e f f}+\mu^{\star}}{\mu_{a}+\mu^{\star}} \quad ; \quad k^{b}=\frac{\mu_{b}}{\mu_{e f f}} \frac{\mu_{e f f}+\mu^{\star}}{\mu_{b}+\mu^{\star}}
$$

$\mu_{a}$ and $\mu_{b}$ are the the single crystal shear moduli. $\mu_{e f f}$ is the shear modulus of the effective medium depending on the estimation that is looked for. $\mu^{\star}$ is the Hill's shear modulus, whose definition depends on the shear and compression moduli of the reference medium supposed isotropic. It is clear, following this relation that the sign of "average" magnetostriction may differ depending on the amplitude of intrinsic parameters that are $\lambda_{100}$ and $\lambda_{111}$. Taking the second order effect into consideration leads to consider that $\lambda_{100}$ and $\lambda_{111}$ are not constant but are stress dependent. A change of sign of picked up magnetostriction (from positive to negative) could explain the change of sensitivity of magnetic behavior at increasing stress.

From a thermodynamic point of view, the above analysis leads to observe that the definition of the mechanical part of the Gibbs free energy given by equation (18) is not unique and corresponds to an assumption where the magnetoelastic term of the Gibbs free energy (usually called magneto-elastic energy) is considered linearly dependent to stress. Indeed the magneto-elastic energy may be presented as a Taylor development of the free energy (including a quadratic magnetic contribution):

$$
\psi_{\alpha}^{\text {magmech }}=-\vec{M}_{\alpha} \otimes \vec{M}_{\alpha}: \mathbb{E}_{\alpha}: \boldsymbol{\sigma}_{\alpha}=M_{s}^{2} \boldsymbol{R}_{\alpha}: \mathbb{E}_{\alpha}: \boldsymbol{\sigma}_{\alpha}
$$

Quantity $M_{s}^{2} \mathbb{E}_{\alpha}$ is often presented as a fourth order magnetostriction tensor, function of three independent constants in case of cubic symmetry. This number reduces to two constants (i.e. $\lambda_{100}$ and $\lambda_{111}$ ) using the (usual) incompressibility condition. We observe the identity: $\left(\boldsymbol{R}_{\alpha}: M_{s}^{2} \mathbb{E}_{\alpha}\right)=\boldsymbol{\epsilon}_{\alpha}^{\mu}$. However a second order development in stress is theoretically possible. It has been proposed by several authors $[28,52]$ to model a phenomenon known as morphic effect. Indeed, using a higher order makes it possible to explain the evolution of the stiffness constants of some materials with the direction of magnetization (nickel [28]). The introduction of a second order stress term leads consequently 
to dependance of compressibility and shear moduli to the magnetic state of the medium. This phenomenon thus contributes in a minor way to the $\Delta E$ effect, mainly related to the evolution of the magnetic domain configuration under stress [30]. This higher order term also allows, as noticed by [52] to make the magnetostrictive "constants" stress dependent. Such a second order development has been previously employed by [27, 48, 53] for strain (Helmholtz free energy was addressed) in macroscopic models. Finally, the magneto-elastic energy is written using a sixth order tensor $\mathcal{E}_{\alpha}\left(M_{s}^{2} \mathcal{E}_{\alpha}\right.$ is the morphic tensor $)$ following:

$$
\begin{gathered}
\psi_{\alpha}^{\text {magmech }}=-\vec{M}_{\alpha} \otimes \vec{M}_{\alpha}: \mathbb{E}_{\alpha}: \boldsymbol{\sigma}_{\alpha}-\frac{1}{2} \boldsymbol{\sigma}_{\alpha}:\left(\vec{M}_{\alpha} \otimes \vec{M}_{\alpha}: \mathcal{E}_{\alpha}\right): \boldsymbol{\sigma}_{\alpha} \\
\psi_{\alpha}^{\text {magmech }}=-M_{s}^{2} \boldsymbol{R}_{\alpha}:\left(\mathbb{E}_{\alpha}: \boldsymbol{\sigma}_{\alpha}+\frac{1}{2} \boldsymbol{\sigma}_{\alpha}: \mathcal{E}_{\alpha}: \boldsymbol{\sigma}_{\alpha}\right)
\end{gathered}
$$

The Gibbs free energy density is now written inside a domain and for a constant as:

$g_{\alpha}=-\frac{1}{2} \boldsymbol{\sigma}_{\alpha}: \mathbb{C}_{\alpha}^{-1}: \boldsymbol{\sigma}_{\alpha}-M_{s}^{2} \boldsymbol{R}_{\alpha}:\left(\mathbb{E}_{\alpha}: \boldsymbol{\sigma}_{\alpha}+\frac{1}{2} \boldsymbol{\sigma}_{\alpha}: \mathcal{E}_{\alpha}: \boldsymbol{\sigma}_{\alpha}\right)+K_{1}\left(\gamma_{1}^{2} \gamma_{2}^{2}+\gamma_{1}^{2} \gamma_{3}^{2}+\gamma_{2}^{2} \gamma_{2}^{2}\right)+K_{2}\left(\gamma_{1}^{2} \gamma_{2}^{2} \gamma_{3}^{2}\right)-\mu_{0} \vec{H}_{\alpha} \cdot \vec{M}_{\alpha}$

This formulation has two consequences:

- Magnetostriction includes now a stress-dependent term. Indeed, total deformation is obtained as the derivation of the Gibbs free energy with respect to stress

$$
\boldsymbol{\epsilon}_{\alpha}=-\frac{\partial g_{\alpha}}{\partial \boldsymbol{\sigma}_{\alpha}}=\mathbb{C}_{\alpha}^{-1}: \boldsymbol{\sigma}_{\alpha}+M_{s}^{2} \boldsymbol{R}_{\alpha}:\left[\mathbb{E}_{\alpha}+\mathcal{E}_{\alpha}: \boldsymbol{\sigma}_{\alpha}\right]
$$

The deformation is separated in elastic and magnetostriction terms following

$\boldsymbol{\epsilon}^{e}=\mathbb{C}_{\alpha}^{-1}: \boldsymbol{\sigma}_{\alpha} \quad$ and $\quad \boldsymbol{\epsilon}_{\alpha}^{\prime \mu}=M_{s}^{2} \boldsymbol{R}_{\alpha}:\left[\mathbb{E}_{\alpha}+\mathcal{E}_{\alpha}: \boldsymbol{\sigma}_{\alpha}\right]=\boldsymbol{\epsilon}_{\alpha}^{\mu}+M_{s}^{2} \boldsymbol{R}_{\alpha}: \mathcal{E}_{\alpha}: \boldsymbol{\sigma}_{\alpha}$

We observe that the magnetostriction strain is the composition of the first order magnetostriction strain defined in equation (17) and a second order term linearly stress dependent.

- Stiffness includes now a magnetic-dependent term. Compliance is the composition of the initial compliance and a fourth order tensor related to the orientation of magnetization via the orientation tensor $\boldsymbol{R}_{\alpha}$ :

$$
\mathbb{C}_{\alpha}^{\prime-1}=-\frac{\partial^{2} g_{\alpha}}{\partial \boldsymbol{\sigma}_{\alpha}^{2}}=\mathbb{C}_{\alpha}^{-1}+\boldsymbol{R}_{\alpha}: \mathcal{E}_{\alpha}
$$


This compliance change is called the morphic effect and can be seen as the intrinsic part of the $\Delta E$ effect $^{5}$.

Of course, one strong difficulty if we want this theory to have praticle application is the identification of material parameters associated with the morphic tensor. Indeed the morphic tensor is a sixth-rank tensor and even if cubic symmetry is considered at the domain scale and using the symmetric properties of Cauchy stress and orientation second-rank tensor, the number of independent constants reduces to 9 (126 independent constants in case of triclinic space group material)[28]. This number remains too high to imagine a simple identification and further simplifications are required. It must be noticed that the incompressibility hypothesis (used for the first-order magnetostriction) cannot apply here since stress usually modifies the volume of a material.

The solution that is proposed is to apply an assumption on space group by using an isotropy condition only for this second-order term. The expression of the Gibbs free energy of the second order term can be derived from the work of [52]. After removal of quadratic stress and magnetically independent terms (modifying the elastic compliance), Kraus ${ }^{6}$ obtained a second-order magneto-elastic component $g_{\alpha}^{\prime \prime m a g m e c h}$ in two terms:

$$
g_{\alpha}^{\prime \prime m a g m e c h}=-\frac{1}{2} M_{s}^{2} \boldsymbol{R}_{\alpha}:\left(\boldsymbol{\sigma}_{\alpha}: \mathcal{E}_{\alpha}: \boldsymbol{\sigma}_{\alpha}\right)=g_{\alpha}^{\prime \text { magmech }_{1}}+g_{\alpha}^{\prime \text { magmech }_{2}}
$$

with

$$
g_{\alpha}^{\prime \prime m a g m e c h}{ }_{1}=-\frac{3}{2} \lambda_{s}^{\prime}\left(\boldsymbol{R}_{\alpha}-\frac{1}{3} \boldsymbol{I}\right): \boldsymbol{L}_{\alpha}
$$

and

$$
g_{\alpha}^{\prime \prime m a g m e c h_{2}}=-\frac{3}{2} \lambda_{s}^{\prime \prime}\left(\boldsymbol{R}_{\alpha}-\frac{1}{3} \boldsymbol{I}\right): \boldsymbol{M}_{\alpha}
$$

where $\lambda_{s}^{\prime}$ and $\lambda_{s}$ " are two more magnetostrictive constants responsible of the second-order magnetoelastic effects considering isotropic conditions, $\boldsymbol{I}$ is the identity second-rank tensor. $\boldsymbol{L}_{\alpha}$ and $\boldsymbol{M}_{\alpha}$ are second-rank stress-dependent tensors defined as function of stress components $\sigma_{i j}$ of $\boldsymbol{\sigma}_{\alpha}$ by:

$$
\boldsymbol{L}_{\alpha}=\left(\begin{array}{ccc}
\sigma_{11}^{2}-\sigma_{22} \sigma_{33} & \sigma_{12} \operatorname{tr}\left(\boldsymbol{\sigma}_{\alpha}\right) & \sigma_{13} \operatorname{tr}\left(\boldsymbol{\sigma}_{\alpha}\right) \\
\sigma_{12} \operatorname{tr}\left(\boldsymbol{\sigma}_{\alpha}\right) & \sigma_{22}^{2}-\sigma_{11} \sigma_{33} & \sigma_{23} \operatorname{tr}\left(\boldsymbol{\sigma}_{\alpha}\right) \\
\sigma_{13} \operatorname{tr}\left(\boldsymbol{\sigma}_{\alpha}\right) & \sigma_{23} \operatorname{tr}\left(\boldsymbol{\sigma}_{\alpha}\right) & \sigma_{33}^{2}-\sigma_{11} \sigma_{22}
\end{array}\right)
$$

and

\footnotetext{
${ }^{5}$ The $\Delta E$ effect defined literaly as a "change of Young modulus" ( $E$ addressing the Young modulus) [7] is the composition of the morphic effect [28] and a reorganization of the domain structure at the upper scale - see [30] for an analytical modeling of this reorganization.

${ }^{6}$ In his paper [52], Kraus calculated the stress-dependent saturation magnetostriction and magnetic-dependent Young modulus of isotropic body. However he did not propose a constitutive behavior.
} 


$$
\boldsymbol{M}_{\alpha}=\left(\begin{array}{ccc}
\sigma_{23}^{2}-\sigma_{22} \sigma_{33} & \sigma_{33} \sigma_{12}-\sigma_{13} \sigma_{23} & \sigma_{22} \sigma_{13}-\sigma_{12} \sigma_{23} \\
\sigma_{33} \sigma_{12}-\sigma_{13} \sigma_{23} & \sigma_{13}^{2}-\sigma_{11} \sigma_{33} & \sigma_{11} \sigma_{23}-\sigma_{12} \sigma_{13} \\
\sigma_{22} \sigma_{13}-\sigma_{12} \sigma_{23} & \sigma_{11} \sigma_{23}-\sigma_{12} \sigma_{13} & \sigma_{12}^{2}-\sigma_{11} \sigma_{22}
\end{array}\right)
$$

where $\operatorname{tr}\left(\boldsymbol{\sigma}_{\alpha}\right)$ indicates the trace of stress tensor at the domain scale. The second order magnetostriction can consequently be derived following:

$$
\boldsymbol{\epsilon}^{\prime \prime \mu}{ }_{\alpha}^{\mu}=M_{s}^{2} \boldsymbol{R}_{\alpha}: \mathcal{E}_{\alpha}: \boldsymbol{\sigma}_{\alpha}=-\frac{\partial g_{\alpha}^{\prime \prime \text { magmech }}}{\partial \boldsymbol{\sigma}_{\alpha}}
$$

leading to the components detailed hereafter:

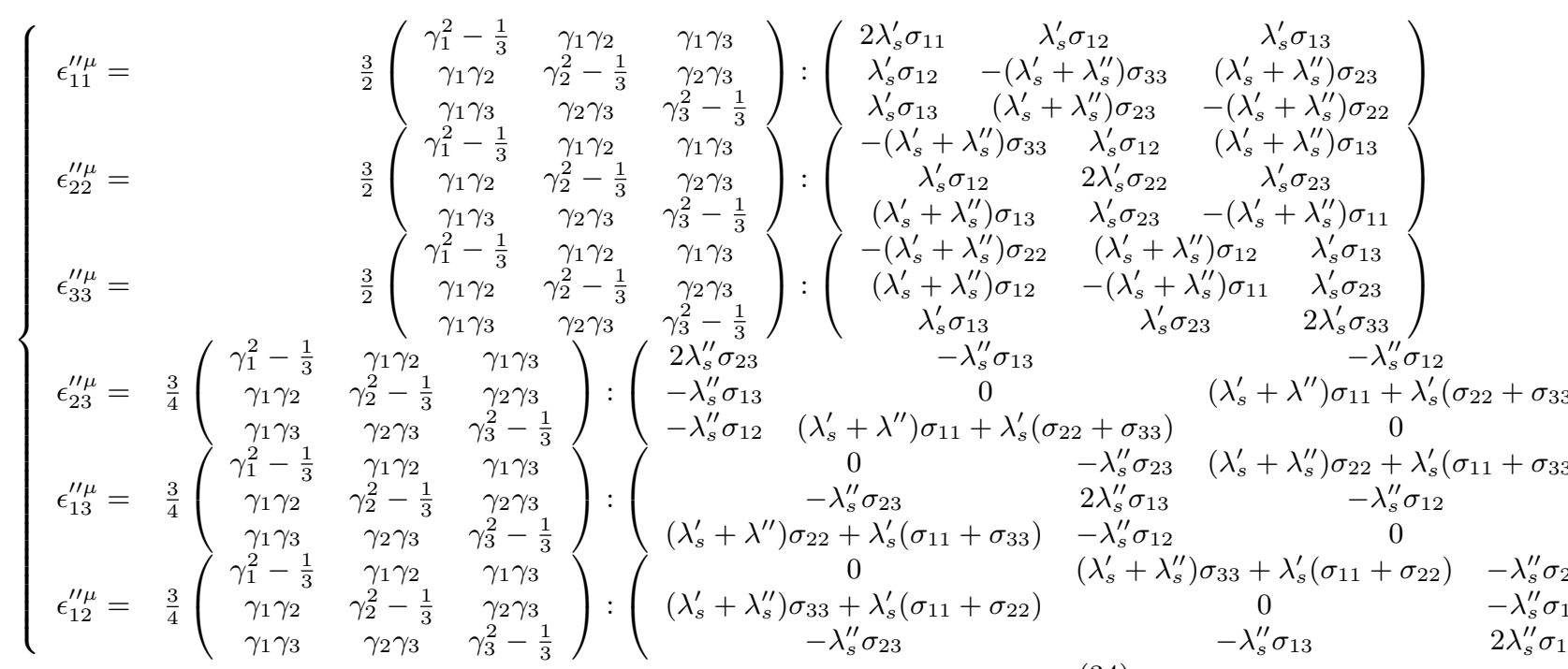

since

$$
\boldsymbol{R}_{\alpha}-\frac{1}{3} \boldsymbol{I}=\left(\begin{array}{ccc}
\gamma_{1}^{2}-\frac{1}{3} & \gamma_{1} \gamma_{2} & \gamma_{1} \gamma_{3} \\
\gamma_{1} \gamma_{2} & \gamma_{2}^{2}-\frac{1}{3} & \gamma_{2} \gamma_{3} \\
\gamma_{1} \gamma_{3} & \gamma_{2} \gamma_{3} & \gamma_{3}^{2}-\frac{1}{3}
\end{array}\right)
$$

\subsection{Constitutive behavior, localization and homogenization}

The objective of the multiscale approach is to calculate, through scale change procedures, the macroscopic magnetization $\vec{M}$ and magnetostriction $\epsilon^{\mu}$ as function of macroscopic magnetic field $\vec{H}$ and stress $\boldsymbol{\sigma}$. Localization (from the macroscale to the domain scale) and homogenization (from domain scale to macroscale) steps are required, involving to apply a constitutive behavior at the local (domain) scale.

$\vec{H}_{\alpha}$ is the magnetic field at the domain scale. $\boldsymbol{\sigma}_{\alpha}$ is the stress tensor at the domain scale. Homogeneous field and stress assumptions at the grain scale (36) 
lead to a stronger simplification of the Gibbs free energy (37) (pure mechanical terms are removed).

$$
\begin{gathered}
\vec{H}_{\alpha}=\vec{H}_{g r} \quad ; \quad \boldsymbol{\sigma}_{\alpha}=\boldsymbol{\sigma}_{g r} \\
g_{\alpha}=-M_{s}^{2} \boldsymbol{R}_{\alpha}:\left(\mathbb{E}_{\alpha}: \boldsymbol{\sigma}_{g r}+\frac{1}{2} \boldsymbol{\sigma}_{g r}: \mathcal{E}_{\alpha}: \boldsymbol{\sigma}_{g r}\right)+K_{1}\left(\gamma_{1}^{2} \gamma_{2}^{2}+\gamma_{1}^{2} \gamma_{3}^{2}+\gamma_{2}^{2} \gamma_{2}^{2}\right)+K_{2}\left(\gamma_{1}^{2} \gamma_{2}^{2} \gamma_{3}^{2}\right)-\mu_{0} \vec{H}_{g r} \cdot \vec{M}_{\alpha}
\end{gathered}
$$

Once the Gibbs free energy $g_{\alpha}$ is known for a given domain $\alpha$ of direction $\vec{\gamma}_{\alpha}=\left[\cos \theta_{\alpha} \sin \phi_{\alpha} ; \sin \theta_{\alpha} \sin \phi_{\alpha} ; \cos \phi_{\alpha}\right]$, its volume fraction $f_{\alpha}(38)$ is calculated according to an explicit Boltzmann-type relation $[18,22]$. This stochastic approach, proposed by other authors $[49,21]$ is relevant since the volume considered is sufficient, assuming that a magnetic domain is much smaller than a representative volume element (considered as a small body immersed into a large closed thermodynamic system)[54]. Numerical results should consequently be compared to experimental results obtained at equilibrium. $A_{s}$ (39) is an adjusting parameter related to the initial magnetic susceptibility $\chi_{0}$ of the material in absence of external or configuration loading $[22,23]$. This calculation is complemented by an energy minimization (40) for the determination of the magnetization direction $\vec{\gamma}_{\alpha}$ (giving the direction cosines ). This formulation is well suited for a single variant and single phase ferromagnetic material (ferromagnetic ferrite or austenite) $)^{7}$.

$$
f_{\alpha}=\frac{\exp \left(-A_{s} g_{\alpha}\right)}{\sum_{\alpha} \exp \left(-A_{s} g_{\alpha}\right)}
$$

with

$$
\begin{gathered}
A_{s}=\frac{3 \chi_{0}}{\mu_{0} M_{s}^{2}} \\
\left\{\theta_{\alpha}, \phi_{\alpha}\right\}=\min \left(g_{\alpha}\right)
\end{gathered}
$$

Assuming that the elastic behavior and the magnetic susceptibility are homogeneous within a grain, the magnetostriction strain and the magnetization of a single crystal are written as the average magnetostriction and magnetization over the domains (41) and (42).

$$
\epsilon_{g r}^{\mu}=<\epsilon_{\alpha}^{\mu}>=\sum_{\alpha} f_{\alpha} \epsilon_{\alpha}^{\mu}
$$

\footnotetext{
${ }^{7} \mathrm{~A}$ more complex formulation has been proposed recently [43] to take a possible phase transformation (ferromagnetic to ferromagnetic, or ferromagnetic to paramagnetic) or the existence of several variants into account. The total number of internal variables strongly depends on the number of grains, phases, variants and number of domain families inside each variant.
} 


$$
\vec{M}_{g r}=<\vec{M}_{\alpha}>=\sum_{\alpha} f_{\alpha} \vec{M}_{\alpha}
$$

This calculation has to be made for each grain of a polycrystalline aggregate. Since grains are not isotropic and disoriented form each other, some localization rules are required to define the stress and magnetic fields at the grain scale as function of macroscopic loadings. An Eshelby approach is applied for the determination of both fields. The orientation of a grain inside the polycrystalline medium is defined by three Euler angles, which are different from one grain to another. The local magnetization and deformation are different from one grain to another. Local magnetic and mechanical loadings $\left(\vec{H}_{g r}\right.$ and $\left.\boldsymbol{\sigma}_{g r}\right)$ are then different from the global loadings $(\vec{H}$ and $\boldsymbol{\sigma})$. Typically the calculation of the local loadings are carried out on each grain through a self-consistent polycrystalline scheme [22], where loadings at the grain scale are derived from the macroscopic loadings, using equations (43) and (44). The gap between local and global quantities defines the demagnetizing field and the residual stress.

$$
\vec{H}_{g r}=\vec{H}+\frac{1}{3+2 \chi_{m}}\left(\vec{M}-\vec{M}_{g r}\right)
$$

$\chi_{m}$ is the secant magnetic susceptibility $\left(\chi_{m}=\|\vec{M}\| /\|\vec{H}\|\right)$,

$$
\boldsymbol{\sigma}_{g r}=\mathbb{B}_{g r}: \boldsymbol{\sigma}+\mathbb{C}^{a c c}:\left(\boldsymbol{\epsilon}^{\mu}-\boldsymbol{\epsilon}_{g r}^{\mu}\right)
$$

with the accommodation stiffness tensor:

$$
\mathbb{C}^{a c c}=\left(\left(\mathbb{C}_{g r}\right)^{-1}+\left(\mathbb{C}^{\star}\right)^{-1}\right)^{-1}
$$

and the stress concentration tensor:

$$
\mathbb{B}_{g r}=\mathbb{C}_{g r}:\left(\mathbb{C}_{g r}+\mathbb{C}^{\star}\right)^{-1}:\left(\mathbb{C}^{0}+\mathbb{C}^{\star}\right):\left(\mathbb{C}^{0}\right)^{-1}
$$

$\mathbb{C}^{\star}=\mathbb{C}^{0}:\left(\left(\mathbb{S}^{E s h}\right)^{-1}-\mathbb{I}\right)$ is the Hill's constraint tensor. $\mathbb{C}^{0}$ is the stiffness tensor of the effective medium. If a self-consistent scheme is chosen, $\mathbb{C}^{0}$ refers to the self-consistent stiffness tensor. $\mathbb{S}^{E s h}$ is the so-called Eshelby tensor, calculated following Mura [55]. The incompatibility operator defines the compatibility effects resulting from the different behaviors between an isolated grain and the surrounding medium [56]. These tensors depend on crystallographic orientation of the considered grain.

Once this self-consistent procedure is converged, the local magnetic and mechanical loadings $\left(\vec{H}_{g r}\right.$ and $\left.\boldsymbol{\sigma}_{g r}\right)$ are determined, leading to the local response of the model $\left(\vec{M}_{g r}, \epsilon_{g r}^{\mu}\right)$.

Once the full knowledge of magnetization and magnetostriction of each grain is obtained, the macroscopic responses $\vec{M}$ and $\epsilon^{\mu}$ of the material are obtained from an averaging operation:

$$
\vec{M}=<\vec{M}_{g r}>
$$




$$
\epsilon_{\mu}=<{ }^{t} \mathbb{B}_{g r}: \epsilon_{g r}^{\mu}>
$$

\section{Comparisons between modeling and experiments}

\subsection{Thermodynamical consistency of experimental results}

We go back to the initial definition of Gibbs free energy (9) and consider the material as homogeneous. The additive decomposition of (macroscopic) elastic and magnetostriction strains apply.

$$
d g=-\left(\boldsymbol{\epsilon}^{e}+\boldsymbol{\epsilon}^{\mu}\right): d \boldsymbol{\sigma}-\mu_{0} \vec{M} \cdot d \vec{H}
$$

The constitutive equations still verify the partial derivatives:

$$
\boldsymbol{\epsilon}^{e}+\boldsymbol{\epsilon}^{\mu}=-\frac{\partial g}{\partial \boldsymbol{\sigma}}
$$

and

$$
\mu_{0} \vec{M}=-\frac{\partial g}{\partial \vec{H}}
$$

These relationships are however applicable at constant stress for the first equation and at constant magnetic field for the second. Since magnetostriction strain and magnetization are now macroscopic, they both depend on magnetic field and stress. The second order derivative of the Gibbs free energy leads to :

$$
\frac{\partial^{2} g}{\partial \boldsymbol{\sigma} \partial \vec{H}}=-\left.\frac{\partial \epsilon^{\mu}}{\partial \vec{H}}\right|_{\sigma}
$$

for the first equation and to:

$$
\frac{\partial^{2} g}{\partial \vec{H} \partial \boldsymbol{\sigma}}=-\left.\mu_{0} \frac{\partial \vec{M}}{\partial \boldsymbol{\sigma}}\right|_{\vec{H}}
$$

for the second equation, where..$\left.\right|_{\boldsymbol{\sigma}}$ and..$\left.\right|_{\vec{H}}$ indicate that relationships are valuable for a constant stress and for a constant magnetic field respectively.

At the energy equilibrium, the two second order partial derivatives are equal leading to the thermodynamic consistency equation (see [51, 37]):

$$
\left.\frac{\partial \boldsymbol{\epsilon}^{\mu}}{\partial \vec{H}}\right|_{\boldsymbol{\sigma}}=\left.\mu_{0} \frac{\partial \vec{M}}{\partial \boldsymbol{\sigma}}\right|_{\vec{H}}
$$

Figure 6a plots the experimental function $\mu_{0} \frac{\partial M}{\partial \sigma}$ get form data plotted in figure 2a. Figure $6 \mathrm{~b}$ plots the experimental function $\frac{\partial \epsilon^{\mu}}{\partial H}$ get form data plotted in figure $4 \mathrm{a}$. The same unit has been used for both functions $(\mathrm{T} / \mathrm{MPa})$ illustrating the same kind of variations and amplitudes (especially at low field and low stress). Furthermore figure $6 \mathrm{c}$ plots the relative difference between 
both functions, corresponding to a thermodynamic relative error (rel. error= $\left.\left(\mu_{0} \frac{\partial M}{\partial \boldsymbol{\sigma}}-\frac{\partial \epsilon^{\mu}}{\partial H}\right) / \max \left(\mu_{0} \frac{\partial M}{\partial \boldsymbol{\sigma}}\right) \times 100\right)$. This error is high where functions exhibit the highest variations but remains lower than $15 \%$. This result confirms that the non-monotonic stress effect observed for the magnetic behavior is related to the change of variation direction of the magnetostriction. An appropriate definition of Gibbs free energy density should therefore enable these two phenomenons to be taken into account simultaneously.

\subsection{Analytical modeling of magnetostrictive behavior including second order effect}

We wish to develop an analytical expression of longitudinal and transversal magnetostriction as function of magnetic field and stress in order to identify the missing magnetostrictive parameters by comparison to experimental data. The analytical modeling we propose is using the following assumptions:

- material is supposed as an agreggate of grains equally distributed over the space and defining a isotropic macroscopic behavior (whatever the behavior);

- cubic space-group is kept for the first order order magnetostriction effect at the grain scale meaning that stress independent magnetostriction is function of parameters $\lambda_{100}$ and $\lambda_{111}$ available in literature for most of materials used for electrical engineering applications;

- isotropic conditions are used for the second order stress effect at the grain scale. The formulation developed at the end of section 3.2 is retained, involving two unknown coefficients $\lambda_{s}^{\prime}$ and $\lambda_{s}^{\prime \prime}$;

- it has been supposed that the magneto-crystalline anisotropy energy does not participate to the evolution of the magnetostriction strain. In such conditions, only the elastic and magnetostatic energies are explicitly considered in the description of the magnetic equilibrium of a domain. As a consequence, this simplified modeling only applies for a magnetization range where domain wall displacement occurs, excluding rotation;

- stress and magnetic field are supposed homogeneous over the material;

- stress is uniaxial and supposed applied in the direction of magnetic field.

A simplified analytical modeling of the $\Delta \mathrm{E}$ effect has been recently proposed [30], using the multiscale model detailed in [22] and in section 3.2. This simplified approach was limited to the situation where no magnetic field was applied, so that the magneto-static energy was not appearing in the definition of the magnetic equilibrium. The integration of magnetic field is necessary and possible but requires a semi- analytical and semi-numerical resolution.

An isotropic polycrystal can be seen as an aggregate of single crystals with random orientation. As argued in [30] and [31], a polycrystal can consequently be considered as a single crystal for which all directions would be easy directions. 
In one domain of such a single crystal, the first order magnetostriction strain tensor can be written (in its own framework):

$$
\boldsymbol{\epsilon}_{\alpha m}^{\mu}=\frac{3}{2}\left(\begin{array}{ccc}
\lambda_{m}\left(\gamma_{1}^{2}-\frac{1}{3}\right) & \lambda_{m} \gamma_{1} \gamma_{2} & \lambda_{m} \gamma_{1} \gamma_{3} \\
\lambda_{m} \gamma_{1} \gamma_{2} & \lambda_{m}\left(\gamma_{2}^{2}-\frac{1}{3}\right) & \lambda_{m} \gamma_{2} \gamma_{3} \\
\lambda_{m} \gamma_{1} \gamma_{3} & \lambda_{m} \gamma_{2} \gamma_{3} & \lambda_{m}\left(\gamma_{3}^{2}-\frac{1}{3}\right)
\end{array}\right)
$$

$\lambda_{m}$ denotes the maximum magnetostriction strain of the considered polycrystal in the direction of the magnetic moment of the considered domain. It can be derived from the first order magnetostrictive constants $\lambda_{100}$ and $\lambda_{111}$ following different assumptions, depending of either or not the magnetization rotation is considered, and depending on the elastic behavior of the domain and the average medium. An analytical calculation of the average magnetostrictive tensor is proposed in [22]. At the magnetic saturation $\lambda_{m}=\lambda_{\text {sat }}$ given by equation (19). If rotation is not considered and for a material exhibiting $<100>$ easy directions, $\lambda_{m}=\frac{2}{5} \lambda_{100} k^{a}$, reducing to $\lambda_{m}=\frac{2}{5} \lambda_{100}$ for homogeneous stress condition.

The magnetization direction of each domain is fixed in space and defined by direction cosines and saturation magnetization (56). Direction cosines are function of spherical angles $\theta$ and $\varphi$ that can be seen as continuous functions varying in the range $(0-2 \pi)$ and $(0-\pi)$ respectively:

$$
\vec{M}_{\alpha}=M_{s}\left(\begin{array}{c}
\gamma_{1} \\
\gamma_{2} \\
\gamma_{3}
\end{array}\right)=M_{s}\left(\begin{array}{c}
\cos \theta \sin \varphi \\
\sin \theta \sin \varphi \\
\cos \varphi
\end{array}\right)
$$

Orthonormal frame $\left(\vec{e}_{1}, \vec{e}_{2}, \vec{e}_{3}\right)$ can be consequently seen as in the macroscopic frame where macroscopic loadings are applied.

We choose direction $\vec{e}_{3}$ as the mechanical and magnetic loading direction. Macroscopic stress tensor and magnetic field vector reduce in:

$$
\boldsymbol{\sigma}=\left(\begin{array}{ccc}
0 & 0 & 0 \\
0 & 0 & 0 \\
0 & 0 & \sigma
\end{array}\right) \quad ; \quad \vec{H}=\left(\begin{array}{c}
0 \\
0 \\
H
\end{array}\right)
$$

The derivation of the maximal magnetostriction uses the same rules for the second-order magnetostriction tensor than for the first order magnetostriction. A $2 / 5$ ratio is applied to isotropic magnetostrictive quantities $\lambda_{s}^{\prime}$ and $\lambda_{s}^{\prime \prime}$. Considering on the other hand the uniaxial condition with applied stress $\sigma_{33}=\sigma$, the second order magnetostriction strain tensor given by equation (34) simplifies in:

$$
\boldsymbol{\epsilon}_{\alpha m}^{\prime \prime \mu}=\frac{3}{5} \sigma\left(\begin{array}{ccc}
-\left(\lambda_{s}^{\prime}+\lambda_{s}^{\prime \prime}\right)\left(\gamma_{2}^{2}-\frac{1}{3}\right) & \left(\lambda_{s}^{\prime}+\lambda_{s}^{\prime \prime}\right)\left(\gamma_{1} \gamma_{2}\right) & \lambda_{s}^{\prime} \gamma_{1} \gamma_{3} \\
\left(\lambda_{s}^{\prime}+\lambda_{s}^{\prime \prime}\right)\left(\gamma_{1} \gamma_{2}\right) & -\left(\lambda_{s}^{\prime}+\lambda_{s}^{\prime \prime}\right)\left(\gamma_{1}^{2}-\frac{1}{3}\right) & \lambda_{s}^{\prime} \gamma_{2} \gamma_{3} \\
\lambda_{s}^{\prime} \gamma_{1} \gamma_{3} & \lambda_{s}^{\prime} \gamma_{2} \gamma_{3} & 2 \lambda_{s}^{\prime}\left(\gamma_{3}^{2}-\frac{1}{3}\right)
\end{array}\right)
$$


The total magnetostriction strain measured in the direction of applied field (and stress) $\epsilon_{\alpha / /}^{\mu}$ is given by the addition of $\epsilon_{\alpha m 33}^{\mu}$ and $\epsilon_{\alpha m 33}^{\prime \prime \mu}$ (59). The total magnetostriction strain measured perpendicularly to the direction of applied field and stress $\epsilon_{\alpha \perp}^{\mu}$ is given by the addition of $\epsilon_{\alpha m 11}^{\mu}$ and $\epsilon_{\alpha m 11}^{\prime \prime \mu}$ averaged with $\epsilon_{\alpha m 22}^{\mu}$ and $\epsilon_{\alpha m 22}^{\prime \prime \mu}$.

$$
\begin{gathered}
\epsilon_{\alpha / /}^{\mu}=\frac{3}{5}\left(\gamma_{3}^{2}-\frac{1}{3}\right)\left(\lambda_{100}+2 \sigma \lambda_{s}^{\prime}\right) \\
\epsilon_{\alpha \perp}^{\mu}=-\frac{3}{10}\left(\gamma_{3}^{2}-\frac{1}{3}\right)\left(\lambda_{100}-\sigma\left(\lambda_{s}^{\prime}+\lambda_{s}^{\prime \prime}\right)\right)
\end{gathered}
$$

These equations can be expressed as function of spherical angles following:

$$
\begin{gathered}
\epsilon_{\alpha / /}^{\mu}=\frac{1}{5}\left(3 \cos ^{2} \varphi-1\right)\left(\lambda_{100}+2 \sigma \lambda_{s}^{\prime}\right) \\
\epsilon_{\alpha \perp}^{\mu}=-\frac{1}{10}\left(3 \cos ^{2} \varphi-1\right)\left(\lambda_{100}-\sigma\left(\lambda_{s}^{\prime}+\lambda_{s}^{\prime \prime}\right)\right)
\end{gathered}
$$

On the other hand the Gibbs free energy of a domain family is calculated following the definitions of section 3.2 and 3.3, and considering the simplified loading and the no-rotation assumption:

$$
g_{\alpha}(H, \sigma)=-\mu_{0} H M_{s} \cos \varphi-\frac{1}{5} \sigma\left(3 \cos ^{2} \varphi-1\right)\left(\lambda_{100}+\sigma \lambda_{s}^{\prime}\right)
$$

Considering homogeneous stiffness, localization operation is avoided. The average magnetostriction is given by:

$$
\boldsymbol{\epsilon}_{m}^{\mu}=\int_{\alpha} f_{\alpha}\left(\boldsymbol{\epsilon}_{m \alpha}^{\mu}+\boldsymbol{\epsilon}_{m \alpha}^{\prime \prime \mu}\right) d \alpha
$$

$f_{\alpha}$ indicates the volume fraction of domain $\alpha$ (see equation (38) in 3.4) calculated using (since $g_{\alpha}$ does not depend on $\theta$ ):

$$
f_{\alpha}=\frac{\exp \left(-A_{s} . g_{\alpha}\right)}{2 \pi \int_{0}^{\pi} \exp \left(-A_{s} . g_{\alpha}\right) \sin \varphi d \varphi}
$$

with (see equation (39) in 3.4):

$$
A_{s}=\frac{3 \chi_{0}}{\mu_{0} M_{s}^{2}}
$$

After few calculations, the longitudinal and transversal magnetostriction strains are obtained:

$$
\begin{gathered}
\epsilon_{m / /}^{\mu}=\int_{0}^{\pi} \frac{\frac{1}{5}\left(3 \cos ^{2} \varphi-1\right)\left(\lambda_{100}+2 \sigma \lambda_{s}^{\prime}\right) \exp \left(A_{s} \mu_{0} H M_{s} \cos \varphi+\frac{1}{5} A_{s} \sigma\left(3 \cos ^{2} \varphi-1\right)\left(\lambda_{100}+\sigma \lambda_{s}^{\prime}\right)\right)}{\int_{0}^{\pi} \exp \left(A_{s} \mu_{0} H M_{s} \cos \varphi+\frac{1}{5} A_{s} \sigma\left(3 \cos ^{2} \varphi-1\right)\left(\lambda_{100}+\sigma \lambda_{s}^{\prime}\right)\right) \sin \varphi d \varphi} \sin \varphi d \varphi \\
\epsilon_{m \perp}^{\mu}=\int_{0}^{\pi} \frac{-\frac{1}{10}\left(3 \cos ^{2} \varphi-1\right)\left(\lambda_{100}-\sigma\left(\lambda_{s}^{\prime}+\lambda_{s}^{\prime \prime}\right)\right) \exp \left(A_{s} \mu_{0} H M_{s} \cos \varphi+\frac{1}{5} A_{s} \sigma\left(3 \cos ^{2} \varphi-1\right)\left(\lambda_{100}+\sigma \lambda_{s}^{\prime}\right)\right)}{\int_{0}^{\pi} \exp \left(A_{s} \mu_{0} H M_{s} \cos \varphi+\frac{1}{5} A_{s} \sigma\left(3 \cos ^{2} \varphi-1\right)\left(\lambda_{100}+\sigma \lambda_{s}^{\prime}\right)\right) \sin \varphi d \varphi} \sin \varphi d \varphi
\end{gathered}
$$

It can be observed that: 
- magnetostriction strains are function of field and stress developing 3D functions and allowing to plot classical $\epsilon^{\mu}(H)$ curves at various stress (magnetostriction behavior) or $\epsilon^{\mu}(\sigma)$ curves at various magnetic field $(\Delta E$ effect);

- the longitudinal magnetostriction strain is function of only one secondorder magnetostriction constant; the identification of this parameter requires to compare numerical results to longitudinal experiments at low field (before rotation).

- the transversal magnetostriction strain is function of both second-order magnetostriction constants; a comparison between numerical results and transversal measurements may allow the second parameter to be identified.

- $\Delta E$ effect curves cannot be used since an initialization of Wheatstone bridges is made during the measurement procedure of magnetostriction behavior under stress. Only relative variations of magnetostriction with magnetic field are relevant.

On the other hand, formula (62) and (63) apply for average magnetization $\vec{M}_{m}$ (rotation not considered) leading to:

$$
\vec{M}_{m}=\int_{\alpha} f_{\alpha} \vec{M}_{\alpha} d \alpha
$$

Component in direction to the applied field $M_{m / /}$ is simply given by:

$M_{m / /}=\int_{0}^{\pi} \frac{M_{s} \cos \varphi \exp \left(A_{s} \mu_{0} H M_{s} \cos \varphi+\frac{1}{5} A_{s} \sigma\left(3 \cos ^{2} \varphi-1\right)\left(\lambda_{100}+\sigma \lambda_{s}^{\prime}\right)\right)}{\int_{0}^{\pi} \exp \left(A_{s} \mu_{0} H M_{s} \cos \varphi+\frac{1}{5} A_{s} \sigma\left(3 \cos ^{2} \varphi-1\right)\left(\lambda_{100}+\sigma \lambda_{s}^{\prime}\right)\right) \sin \varphi d \varphi} \sin \varphi d \varphi$

We are able now to plot longitudinal and transversal magnetostriction as function of magnetization and optimize $\lambda_{s}^{\prime}$ and $\lambda_{s}^{\prime \prime}$ parameters.

\subsection{Experimental identification of morphic parameter}

Different set of $\lambda_{s}^{\prime}$ and $\lambda_{s}^{\prime \prime}$ parameters has been tested. The complexity of the model (even simplified) makes it very difficult to set up an identification procedure by minimization of a residue. Parameters finally chosen are probably not optimal in this sense but satisfy the overall behavior. Table 1 gathers the different parameters finally used for graphical representations. Parameters $\mathrm{M}_{s}$, $\lambda_{100}$ and $\lambda_{111}$ come from literature [6]. Parameter $\chi_{0}$ is corresponding to the initial slope of magnetization curve at the unloaded state. Parameters $\lambda_{s}^{\prime}$ and $\lambda_{s}^{\prime \prime}$ have been optimized by comparison to experimental magnetostriction curves.

Figure 7 plots the magnetic behavior and initial susceptibility as function of stress as given by the simplified model when only the first order magnetoelastic term is considered in the Gibbs free energy. We observe as expected a 
Table 1: Parameters used in the simplified modeling of NO 3wt.\%Si-Fe alloy

\begin{tabular}{|c||c|c|c|c|c|c|}
\hline Parameter & $\mathrm{M}_{s}$ & $\lambda_{100}$ & $\lambda_{111}$ & $\chi_{0}$ & $\lambda_{s}^{\prime}$ & $\lambda_{s}^{\prime \prime}$ \\
\hline Value & $1.61 \times 10^{6}$ & 23.5 & -4.5 & 9600 & -0.295 & 1.180 \\
Unit & $\mathrm{A} / \mathrm{m}$ & $\mathrm{ppm}$ & $\mathrm{ppm}$ & - & $\mathrm{ppm} / \mathrm{MPa}$ & $\mathrm{ppm} / \mathrm{MPa}$ \\
\hline
\end{tabular}

monotonous improvement of magnetic behavior. The associated modeled magnetostriction curves (longitudinal and transversal) are plotted in figure 8. Magnetostriction saturates with increasing stress without sign change, in accordance with the saturation of magnetic behavior without decreasing of susceptibility.

In figure 9 , the taking into account of the second order stress terms leads to a better representation of the magnetic and magnetostrictive behaviors in the range of field and stress studied in the experimental part. Magnetic behavior for $78 \mathrm{MPa}$ tensile stress joins the magnetic behavior at unloaded state. The maximal susceptibility is however obtained for a stress of about $40 \mathrm{MPa}$, higher than the value of $20 \mathrm{MPa}$ observed in the experiments (figure 9b). Magnetostriction curves show the expected change in curvature, directly related to the amplitudes of $\lambda_{s}^{\prime}$ and $\lambda_{s}^{\prime \prime}$ parameters (figures 10a and 10b). The simplified approach does not, however, make it possible to reproduce all the fineness of the experimental behavior. Given the variations observed, we are entitled to question the behavior of this model at higher stress on the one hand, and in compression on the other hand. It is indeed important to observe the limits of the proposed approach.

The effect of wide stress range (from $-500 \mathrm{MPa}$ to $+500 \mathrm{MPa}$ ) on initial susceptibility has been plotted in figure 11a: it is first interesting to observe that modeling using first or second order stress effect leads to a very similar behavior under compressive stress. In case of tensile stress, the susceptibility reaches three times its nominal value when the first order stress effect is used: this result was foreseen but is of course not in accordance with experimental results. The second order stress formulation brings to null susceptibility after $200 \mathrm{MPa}$. Indeed the quadratic stress form governs the Gibbs free energy at high stress, which leads to a global effect close to a compression effect on magnetic behavior. Even if high stress range has not been tested, the observed trends in figure $3 \mathrm{~b}$ bring to a saturation of susceptibility at a level of the same order of magnitude as the susceptibility at zero stress. Figure $11 \mathrm{~b}$ reports the magnetostriction deformation only due to stress (at zero applied magnetic field and zero magnetization), highlighting the so-called $\Delta \mathrm{E}$ effect [7]. Longitudinal and transversal deformations are plotted. A saturation of $\Delta \mathrm{E}$ effect is observed in tension and compression for both directions when only the first order stress term is considered. When the second order expression is employed, saturation disappears and curves reach a constant slope (positive slope $\zeta_{/ /}$along the tensile direction; negative slope $\zeta_{\perp}$ along the direction transversal to the applied stress). Indeed, slopes $\zeta_{/ /}$and $\zeta_{\perp}$ are directly related to the second derivative of the Gibbs free 
energy and to isotropic parameters $\lambda_{s}^{\prime}$ and $\lambda_{s}^{\prime \prime}$ highlighting the so-called morphic effect [40]:

$$
\zeta_{/ /}=\frac{d \epsilon_{m / /}^{\mu}}{d \sigma}=-\frac{2}{5} \lambda_{s}^{\prime} \quad \text { and } \quad \zeta_{\perp}=\frac{d \epsilon_{m \perp}^{\mu}}{d \sigma}=\frac{1}{10}\left(\lambda_{s}^{\prime}+\lambda_{s}^{\prime \prime}\right)
$$

These values correspond to an equivalent stiffness of about $8500 \mathrm{GPa}$ (to be compared to a typical Young modulus of $200 \mathrm{GPa}$ ), not detectable by a classical tensile stress / strain experiment. 


\subsection{Comparison between experiments and multiscale modeling}

The application of the full multiscale constitutive model of NO $3 \% \mathrm{Si}-\mathrm{Fe}-\mathrm{Si}$ alloy is presented in this section with or without considering the second order magnetoelastic term in the Gibbs free energy expression. Parameters used, mixing literature values and parameters fitted using the simplified modeling $\left(\chi_{0}, \lambda_{s}^{\prime}\right.$ and $\left.\lambda_{s}^{\prime \prime}\right)$ are given in table 2. An orientation data file containing 396 orientations representative of the crystallographic texture of the material has been used for the modeling (figure 12). Data are issued from previous electron back-scattered diffraction (EBSD) measurements [32, 23].

Table 2: Parameters used in the mutliscale modeling of NO 3wt.\%Si-Fe alloy

\begin{tabular}{|c||c|c|c|c|c|c|}
\hline Parameter & $\mathrm{M}_{s}$ & $K_{1} ; K_{2}$ & $\lambda_{100} ; \lambda_{111}$ & $\chi_{0}$ & $\lambda_{s}^{\prime} ; \lambda_{s}^{\prime \prime}$ & $C_{11} ; C_{12} ; C_{44}$ \\
\hline Value & $1.61 \times 10^{6}$ & $38 ; 0$ & $23.5 ;-4.5$ & 9600 & $-0.295 ; 1.180$ & $202 ; 122 ; 229$ \\
Unit & $\mathrm{A} / \mathrm{m}$ & $\mathrm{kJ} / \mathrm{m}^{3}$ & $\mathrm{ppm}$ & - & $\mathrm{ppm} / \mathrm{MPa}$ & $\mathrm{GPa}$ \\
\hline
\end{tabular}

Figures 13a and 13b show the results of multiscale modeling of magnetic behavior when the material is submitted to a uniaxial stress applied along the transversal direction (TD). The magnetic field is also applied along TD. As already noticed in [23], the model tends to overestimate the magnetization at low field whatever the retained magneto-elastic description. This is attributed to the fact that intragranular heterogeneities and local demagnetizing effects are insufficiently taken into account by the modeling. Trends observed with the simplified modeling remain of course unchanged. Taking account of rotation mechanism and crystallographic texture does not change the global variations. The modeling using only the first order stress term provides a constant improvement of the magnetic behavior at increasing stress levels. When the second order stress term is considered, the overestimation of the magnetization for a given magnetic field can still be noticed, but the effect of stress on the behavior is satisfactorily described (figure 14). This point is clearly highlighted by the plot of initial susceptibilty provided by the two modelings in figure 14. The nonmonotonic dependence of the magnetic susceptibility to the applied uniaxial stress is appropriately described when the second order magneto-elastic term is considered. Furthermore, general trends are correctly rendered by the new modeling for the description of the magnetostriction strain as reported in figure $15 \mathrm{a}$ and $15 \mathrm{~b}$, meeting the trends observed with the simplified modeling.

Extension of the modeling to higher stress magnitude seems however not completely satisfactory as illustrated in figure 16 . The effect of wide stress range (from $-500 \mathrm{MPa}$ to $+500 \mathrm{MPa}$ ) on initial susceptibility has been plotted in figure $16 \mathrm{a}$ considering or not considering the second order stress effect. Figure 16b reports on the other hand the longitudinal and transversal $\Delta \mathrm{E}$ effect for both modeling. We observe first that these results elicit the same global comments as with the simplified model. Some particular points can be highlighted: 
- modeling using first or second order stress effect leads to a behavior under compressive stress different from that observed with the simplified modeling: susceptibility obtained with the first order stress modeling reaches a saturation value close to 2000 although this value reaches 0 for the second order stress modeling. This difference can be assigned to rotation mechanism.

- the initial susceptibility modeled when the first order stress effect is considered reach a high value (two times the nominal value) before to slowly decrease with increasing tensile stress. It is another illustration of rotation effect corresponding to Villari reversal. The introduction of second order stress term completely hides this mechanism.

- $\Delta \mathrm{E}$ effect magnitude reported in figure $16 \mathrm{~b}$ is about 2.5 times the magnitudes observed in case of simplified modeling. This is the result of magnetization rotation too, that leads to a transformation of factors $2 / 5$ and $-1 / 10$ in equations (68) into factors 1 and $-1 / 2$.

As discussed in the case of simplified modeling, the initial susceptibility would tend to saturate at a level close to the initial susceptibility observed at zero stress. This trend is not rendered by the modeling, restricting the validity range of the present modeling to the range of experiments reported in the paper.

\section{Conclusion}

The objective of this paper was to be able to better account of the nonmonotonic evolution of the magnetic behavior under tensile stress by an adequate modeling, in a range where the Villari reversal is still not significant. Taking into account higher order stress terms in the expression of the Gibbs free energy seems to be the key. On the one hand, it makes it possible to account for the non-monotonic effect and, on the other hand, to model in a realistic way the corresponding evolution of the longitudinal and transversal magnetostrictive behaviors. In addition, a procedure using a simplified semi-analytical model was developed to identify two new magnetostrictive parameters. The description, however, remains limited to an isotropic framework for the second-order stress term, which can lead to some uncontrolled phenomena at this stage. Applying the model to higher stress levels also clearly shows the limitations of the proposed approach. In addition to a complete identification of second-order terms within the framework of cubic symmetry (using the same kind of set-up and single crystals cut along several crystallographic directions), the search for functions of high order stress invariants may be a preferred path for the future, leading however to a more complex dependence of the magnetic behavior to mechanical stresses. 
[1] FeCoProGen project, "FeCo based magnetic alloys with high strength and minimized iron losses, for new high speed aerospace generator", Grant agreement ID: 620142 (2013-2015), EU FP7 - Cleansky.

[2] G. Qiao, G. Liu, Z. Shi, Y. Wang, S. Ma and T. Lim, "A review of electromechanical actuators for More/All Electric aircraft systems", Journal of Mechanical Engineering Science, 23222 (2018), pp.4128-4151.

[3] V. Madonna, P. Giangrande and M. Galea, "Electrical Power Generation in Aircraft: Review, Challenges, and Opportunities", IEEE Transactions on Transportation Electrification, 43 (2018), pp.646-659.

[4] P. Arumugam, Z.Xu, A. La Rocca, G. Vakil, M. Dickinson, E.Amankwah, T. Hamiti, S. Bozhko, Chris Gerada and Stephen J. Pickering, "High-Speed Solid Rotor Permanent Magnet Machines: Concept and Design", IEEE Transactions on Transportation Electrification, 23 (2016), pp 391-400.

[5] M. Rekik, O. Hubert, L. Daniel, "Influence of a multiaxial stress on the reversible and irreversible magnetic behaviour of a $3 \% \mathrm{Si}-\mathrm{Fe}$ alloy", International Journal of Applied Electromagnetics and Mechanics, 44 3-4 (2014), pp.301-315.

[6] Bozorth R.M., "Ferromagnetism", ed. D. Van Norstand, N.Y. 1951.

[7] Cullity B.D., "Introduction to magnetic materials", ed. Addison-Wesley, N.Y. 1972.

[8] M.J. Sablik, H. Kwun, G.L. Burkhardt, D.C. Jiles, "Model for the effect of tensile and compressive stress on ferromagnetic hysteresis", Journal of Applied Physics, 61 (1987), pp.3799-3801.

[9] M.J. Sablik and D.C. Jiles, "Coupled Magnetoelastic Theory of Magnetic and Magnetostrictive Hysteresis", IEEE Transactions on Magnetics, 293 (1993), pp.2113-2123.

[10] C. Schneider, "Effect of stress on the shape of ferromagnetic hysteresis loops", Journal of Applied Physics, 97 (2005), 10E503.

[11] A. Belahcen and A. Arkkio, "Locally coupled magneto-mechanical model of electrical steel", COMPEL, 27 (2008) pp.1451-1462.

[12] D. Singh, F. Martin, P. Rasilo and A. Belahcen, "Magnetomechanical Model for Hysteresis in Electrical Steel Sheet," IEEE Transactions on Magnetics, 52, 11 (2016) pp.1-9, 7301109.

[13] A.A. Abdallh, L. Dupré, "The influence of magnetic material degradation on the optimal design parameters of electromagnetic devices", IEEE Trans. Mag. (2017) 
[14] M.J. Sablik, L.A. Riley, G.L. Burkhardt, H. Kwun, P.Y. Cannell, K.T. Watts, R.A. Langman, "Micromagnetic model for the influence of biaxial stress on hysteretic magnetic properties", Journal of Applied Physics, 75 (1994), pp.5673-5675.

[15] J. Pearson, P. T. Squire, M. G. Maylin, and J. G. Gore, "Biaxial stress effects on the magnetic properties of pure iron", IEEE Transactions on Magnetics, 365 (2000), pp. 3251-3523.

[16] O. Hubert, L. Daniel, "Energetical and multiscale approaches for the definition of an equivalent stress for magneto-elastic couplings", Journal of Magnetism and Magnetic Materials, 323, (2011), pp.1766-1781.

[17] W.D. Armstrong, "Magnetization and magnetostriction processes in $\mathrm{Tb}(0.27-0.30) \mathrm{Dy}(0.73-0.70) \mathrm{Fe}(1.9-2.0)$ ". Journal of Applied Physics, 81 (1997), pp.2321-2326.

[18] N. Buiron, L. Hirsinger and R. Billardon, "A multiscale model for magnetoelastic couplings", Journal de Physique IV, 9 (1999), pp.187-196.

[19] W.D. Armstrong, "A directional magnetization potential based model of magnetoelastic hysteresis", Journal of Applied Physics, 91 (2002), pp.22022210 .

[20] A. De Simone, R. James, "A constrained theory of magnetoelasticity", Journal of Mechanics and Physics of Solids, 50 (2002), pp.283-320.

[21] R.C. Smith, S. Seelecke, M. Dapino, Z. Ounaies, "A unified framework for modeling hysteresis in ferroic materials", Journal of the Mechanics and Physics of Solids, 54 (2006), pp.46-85.

[22] L. Daniel, O. Hubert, N. Buiron and R. Billardon, "Reversible magnetoelastic behavior: a multiscale approach", Journal of the Mechanics and Physics of Solids, 56 (2008), pp.1018-1042.

[23] L. Daniel, M. Rekik, O. Hubert, "A multiscale model for magneto-elastic behaviour including hysteresis effects", Archive of Applied Mechanics, 84, 9 (2014), pp.1307-1323.

[24] L. Bernard, X. Mininger, L. Daniel, G. Krebs, F. Bouillault, M. Gabsi, "Effect of stress on switched reluctance motors: a magneto-elastic finiteelement approach based on multiscale constitutive laws", IEEE Transactions on Magnetics, 47 issue 9, (2011), pp.2171-2178.

[25] O. Perevertov, "Influence of the applied elastic tensile and compressive stress on the hysteresis curves of Fe-3\%Si non-oriented steel", Journal of Magnetism and Magnetic Materials, 428 (2017), pp.223-228

[26] O. Hubert, S. Lazreg, "Two phase modeling of the influence of plastic strain on the magnetic and magnetostrictive behaviors of ferromagnetic materials", Journal of Magnetism and Magnetic Materials, 4242 (2017), pp.421-442. 
[27] U. Aydin, P. Rasilo, F. Martin, D. Singh, L. Daniel, A. Belahcen, M. Rekik, O. Hubert, R. Kouhia, A. Arkkio, "Magneto-mechanical modeling of electrical steel sheets", Journal of Magnetism and Magnetic Materials, 439 (2017), pp.82-90.

[28] W.P. Mason, "A Phenomenological Derivation of the First- and SecondOrder Magnetostriction and Morphic Effects for a Nickel Crystal", Physical Review, 825 (1951), pp.715-723.

[29] E. Du Trémolet de Lacheisserie, "magnetoelastic properties of amorphous alloys", Journal of Magnetism and Magnetic Materials, 25 (1982), pp.251270 .

[30] L. Daniel, O. Hubert, "An analytical model for the $\Delta \mathrm{E}$ effect in magnetic materials", European Physical Journal - Applied Physics, 45 (2009) 31101.

[31] O. Hubert, X. Milhet, P. Gadaud, M. Tatat, P.-O. Renault, C. Coupeau, "Modeling of Young's modulus variations with temperature of $\mathrm{Ni}$ and oxidized Ni using a magneto-mechanical approach", Materials Science \& Engineering, A 633 (2015) pp.76-91.

[32] O. Hubert, L. Daniel, R. Billardon, "Experimental analysis of the magnetoelastic anisotropy of a non-oriented silicon iron alloy", Journal of Magnetism and Magnetic Materials, 254-255 (2003), pp.352-354.

[33] C. Gourdin, "Identification et modlisation du comportement lectromagntique de structures ferromagntiques", phD thesis, Université Pierre et Marie Curie, France (1998).

[34] L. Daniel, O. Hubert, F. Ossart and R. Billardon, "Experimental analysis and multiscale modelling of the anisotropic mechanical and magnetostrictive behaviours of electrical steels", Journal de Physique IV France, 105, 3 (2003), pp.247-254.

[35] O. Hubert and L. Daniel, "Effect of plastic straining on magnetostriction of ferromagnetic polycrystals - experiments and multiscale modeling", Journal of Magnetism and Magnetic Materials, 304, 9 (2006), pp.489-491.

[36] O. Hubert and K.J. Rizzo, "Anhysteretic and dynamic piezomagnetic behavior of a low carbon steel", Journal of Magnetism and Magnetic Materials, 32020 (2008), pp. 979-982.

[37] C. Bormio-Nunes, O. Hubert, "Piezomagnetic behavior of Fe-Al-B alloys", Journal of Magnetism and Magnetic Materials, 39311 (2015), pp.404-418.

[38] K.J. Rizzo, O. Hubert and L.Daniel, "A multiscale model for piezomagnetic behavior", European Journal of Electrical Engineering, 124 (2009), pp.525540. 
[39] S. Lazreg and O. Hubert, "A multidomain modeling of the magnetoelastic behavior for nondestructive evaluation", Journal of Applied Physics, 109 (2011), 07E508

[40] E. Du Trémolet de Lacheisserie, Magnetostriction, Theory and Applications of Magnetoelasticity, CRC Press, Boca Raton, 1993.

[41] O. Hubert, "Influence of biaxial stresses on the magnetic behaviour of an iron-cobalt sheet - Experiments and modelling", Przedglad Elektrotecniczny, R.83 (2007), pp.70-77.

[42] A. Maynadier, D. Depriester, K. Lavernhe-Taillard and O.Hubert, "Thermo-mechanical description of phase transformation in Ni-Ti Shape Memory Alloy", Procedia Engineering, 10 (2011), pp.2214-2219.

[43] O Hubert, K Lavernhe-Taillard, M.D. Fall, X. Chang, M. Savary, O Sevestre, "Multiscale Approach for the Modeling of Chemo-MagnetoThermo-Mechanical Couplings - Reversible Framework", Materials Science Forum, 941 (2018), pp.2290-2295.

[44] M.D. Fall, O.Hubert, F. Mazaleyrat, K. Lavernhe-Taillard, A. Pasko, "Multiscale Modeling of Magnetic Shape Memory Alloys: Application to NiMnGa Single Crystal", IEEE Transactions on Magnetics, 525 (2016), pp.1-4.

[45] L.Néel, "Les lois de laimantation et de la subdivision en domaines élémentaires dun monocristal de fer", Journal de Physique et le Radium, 5 (1944), pp.241-251.

[46] O. Hubert, L. Daniel, "Multiscale modelling of magneto-mechanical behaviour of Grain Oriented Silicon Steels", Journal of Magnetism and Magnetic Materials, 320, (2008), pp.1412-1422.

[47] J. Y. Gauthier, C. Lexcellent, A. Hubert, J. Abadie, N. Chaillet, "Modeling rearrangement process of martensite platelets in a magnetic shape memory alloy $\mathrm{Ni}_{2}$ MnGa single crystal under magnetic field and (or) stress action", Journal of Intelligent Materials Systems and Structures, 183 (2007), pp.289299.

[48] S.V. Kankanala, N. Triantafyllidis, "On finitely strained magnetorheological elastomers", Journal of the Mechanics and Physics of Solids, 52 (2004), pp.2869-2908.

[49] S. Chikazumi, "Physics of Ferromagnetism", second ed., Oxford University Press, 1997.

[50] A. Hubert, R.Schäfer, "Magnetic domains", Springer Berlin Heidelberg, 1998. 
[51] C. Gourdin, L. Hirsinger, G. Barbier, R. Billardon, "Experimental identification of the coupling between the anhysteretic magnetic and magnetostrictive behaviours", Journal of Magnetism and Magnetic Materials 177-181 (1998), pp. 201-202.

[52] L. Kraus, "On the Stress Dependence of the Saturation Magnetostriction in Amorphous Alloys", Physic status solidi (a) 109 (1988), pp.71-74.

[53] K. Fonteyn, A. Belahcen, R. Kouhia, P. Rasilo, and A. Arkkio, FEM for Directly Coupled Magneto-Mechanical Phenomena in Electrical Machines, IEEE Transactions on Magnetics, 468 (2010), pp.2923-2926.

[54] A. Sommerfeld, Thermodynamics and Statistical Physics, Academic Press, N.Y., 1955.

[55] T. Mura, "Micromechanics of Defects in Solids", ed. Martinus Nijhoff Publishers, Dordrecht, MA, 1982.

[56] R. Hill, "Continuum micro-mechanics of elastoplastic polycrystals", Journal of the Mechanics and Physics of Solids 13 (1965) pp.89-101. 


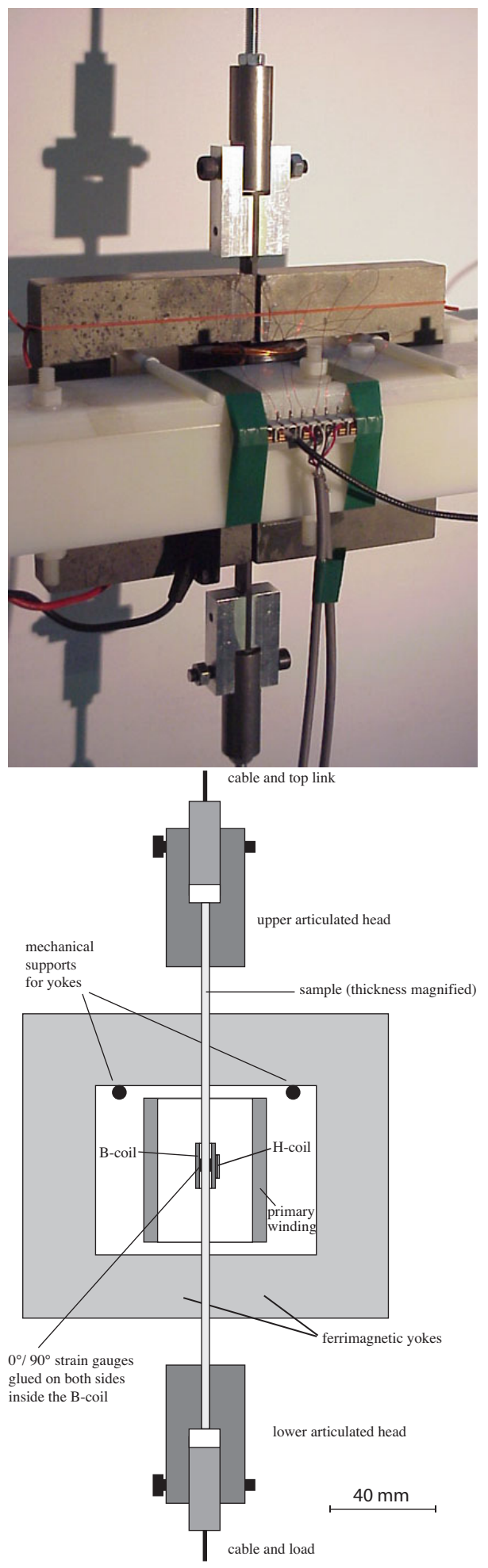

Figure 1: Apparatus for measurement of magnetic behavior under applied stress - with articulated heads 

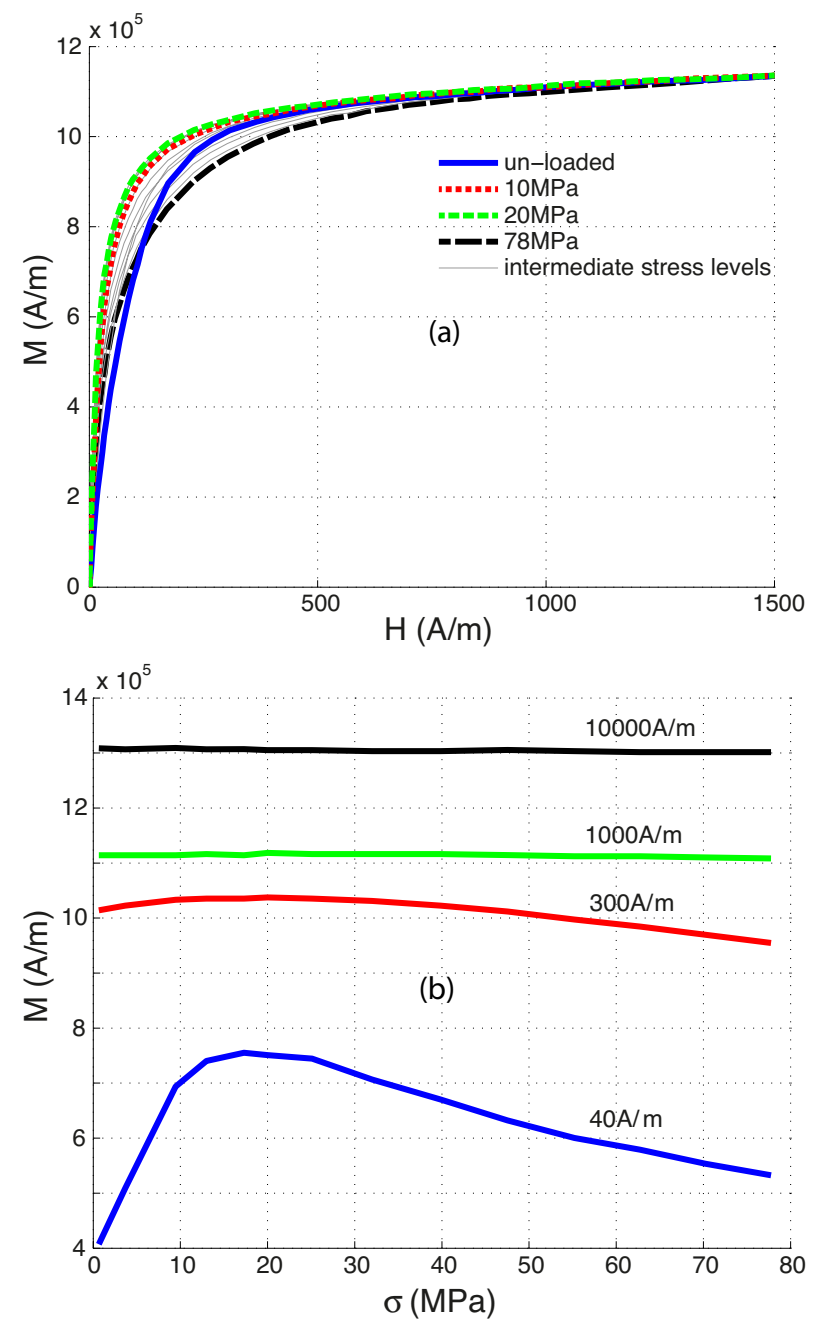

Figure 2: Effect of uniaxial stress on magnetization behavior of NO 3wt.\%Si-Fe: (a) magnetization at constant stress; (b) magnetization at constant magnetic field. 

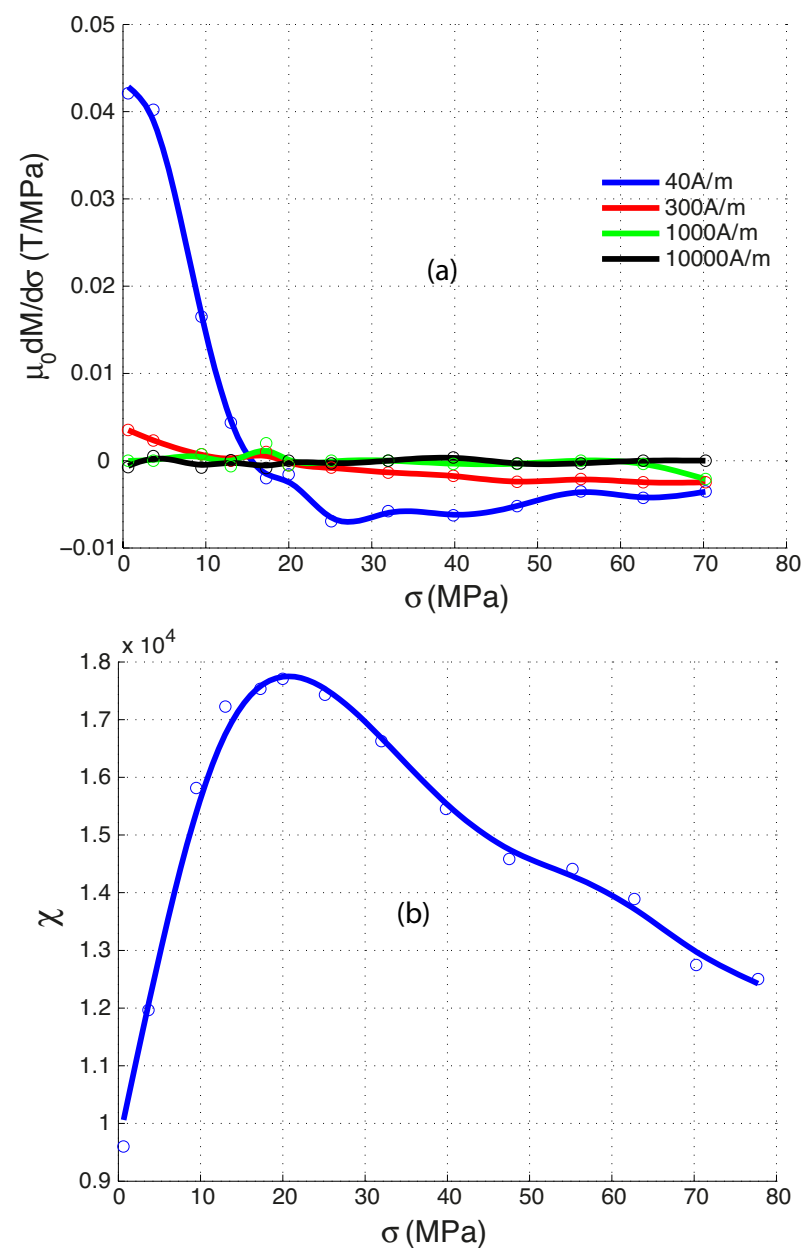

Figure 3: Effect of uniaxial stress on magnetization behavior of NO 3wt.\%Si-Fe: (a) sensitivity of magnetization to stress ( $\mu_{0}$ : vacuum permeability) at constant magnetic field; (b) initial anhysteretic susceptibility $\chi$ as function of stress. 

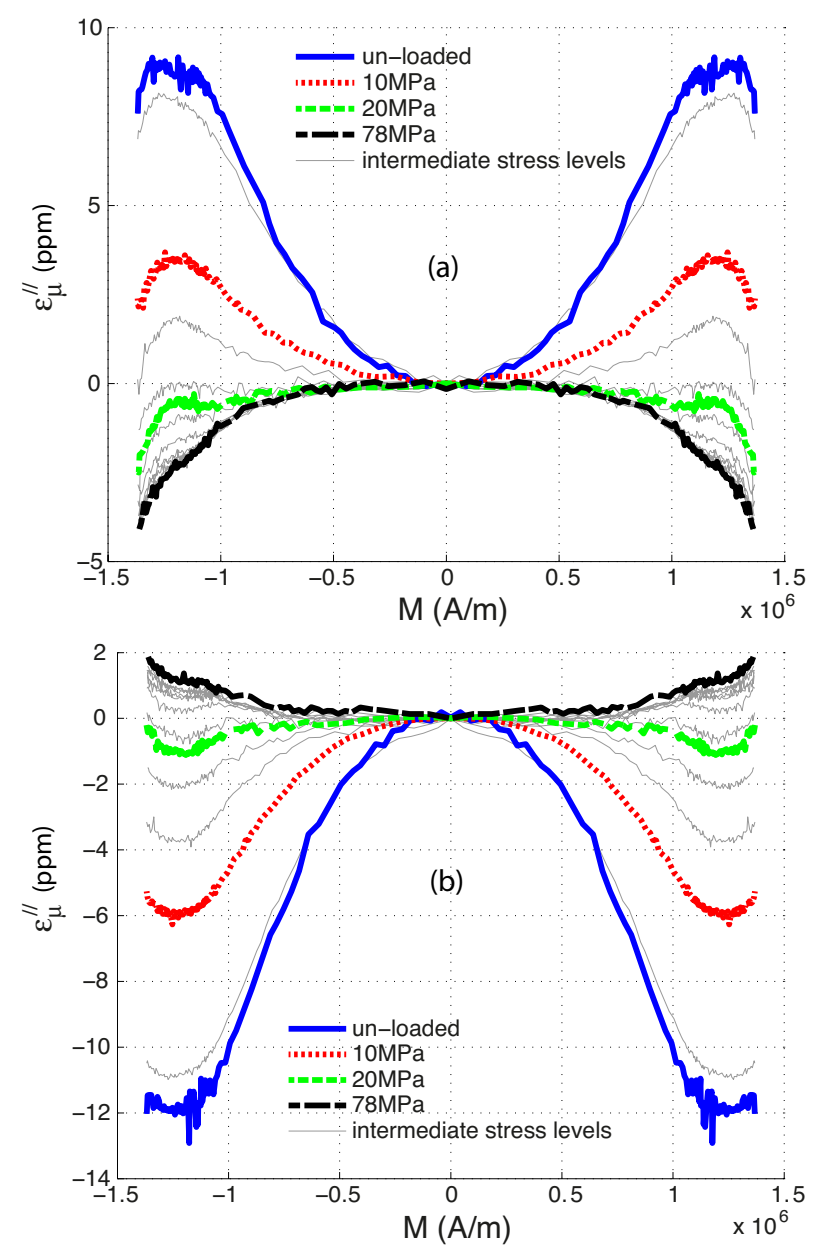

Figure 4: Effect of uniaxial stress on magnetostriction behavior of NO 3wt.\%Si-Fe: (a) longitudinal magnetostriction; (b) transversal magnetostriction. 


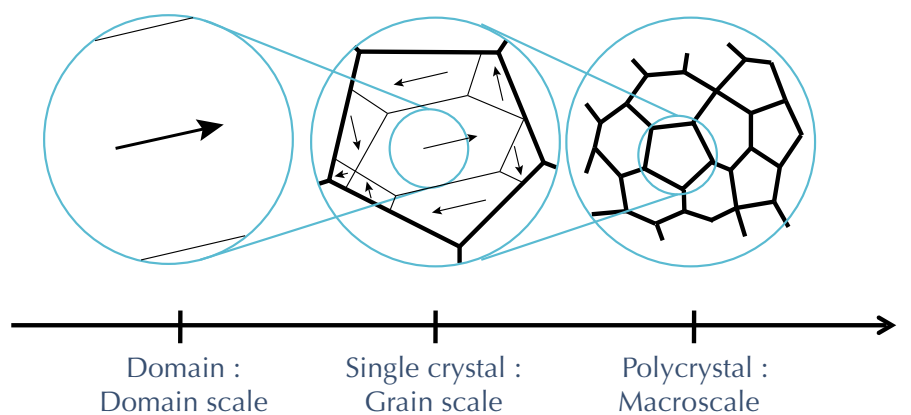

Domain scale Grain scale Macroscale

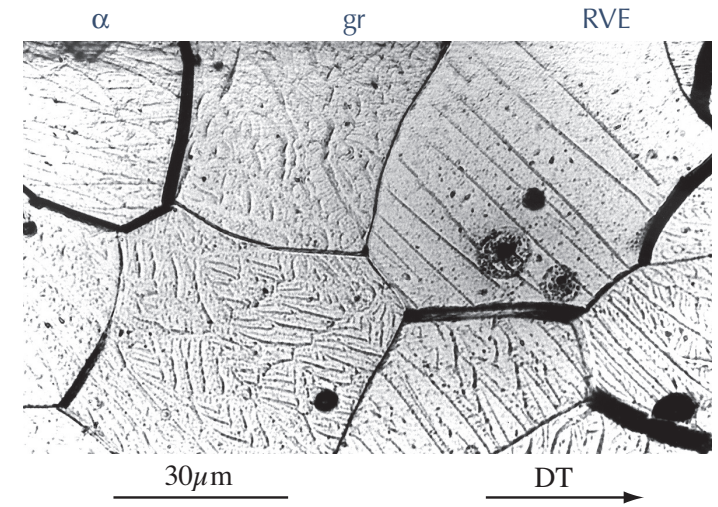

Figure 5: Detail of scales involved in the modeling approach (arrows address local magnetization vectors); optical observation of the magnetic domains structure inside the grains of a polycrystalline NO $3 \% \mathrm{Si}-\mathrm{Fe}$ alloy - Bitter technique. 

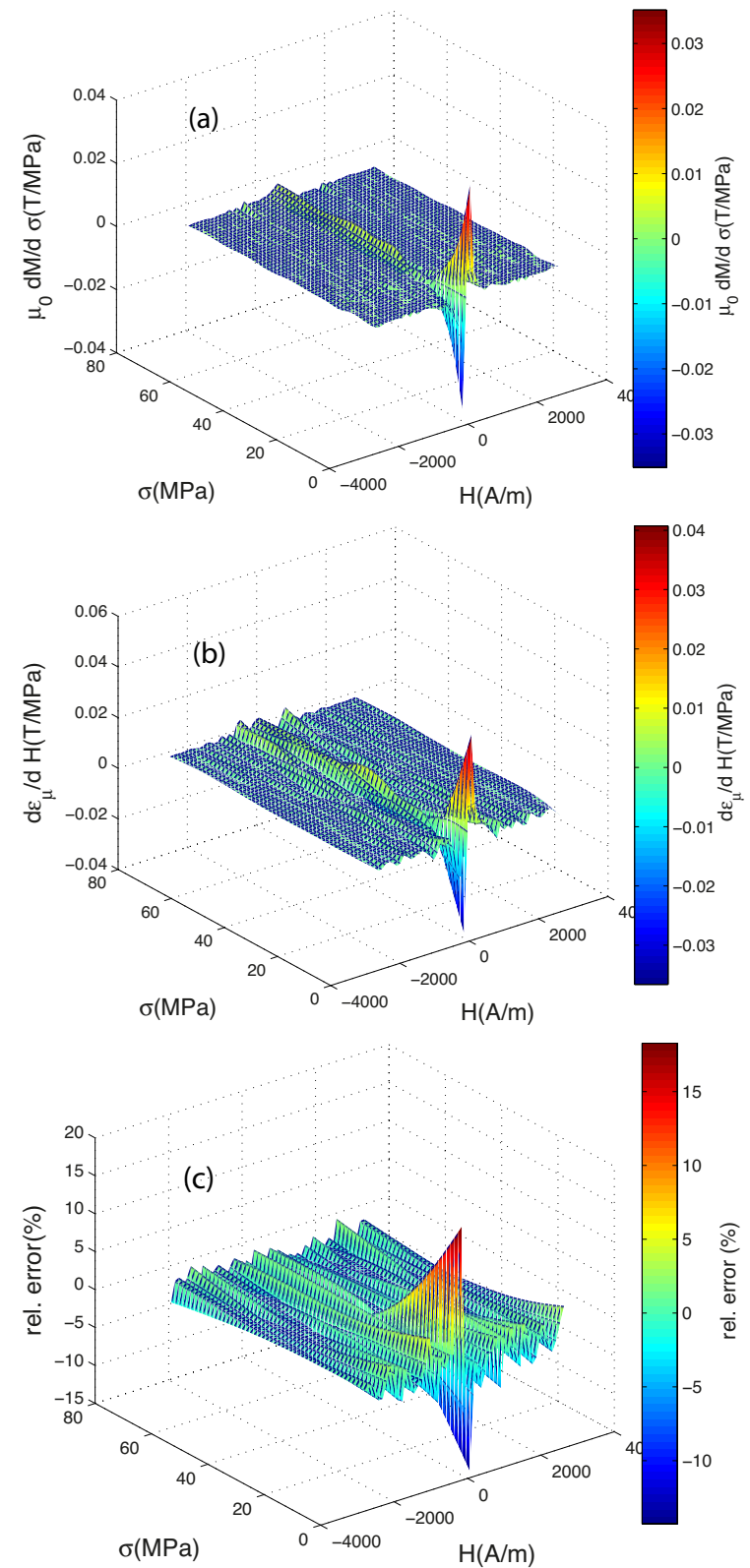

Figure 6: Verification of thermodynamic consistency equation (54) from experimental data obtained with NO 3wt.\%Si-Fe alloy: (a) plot of $\mu_{0} \frac{\partial M}{\partial \sigma}$; (b) plot of $\frac{\partial \epsilon^{\mu}}{\partial H}$; (c) relative difference between two functions - as function of stress and magnetic field. 

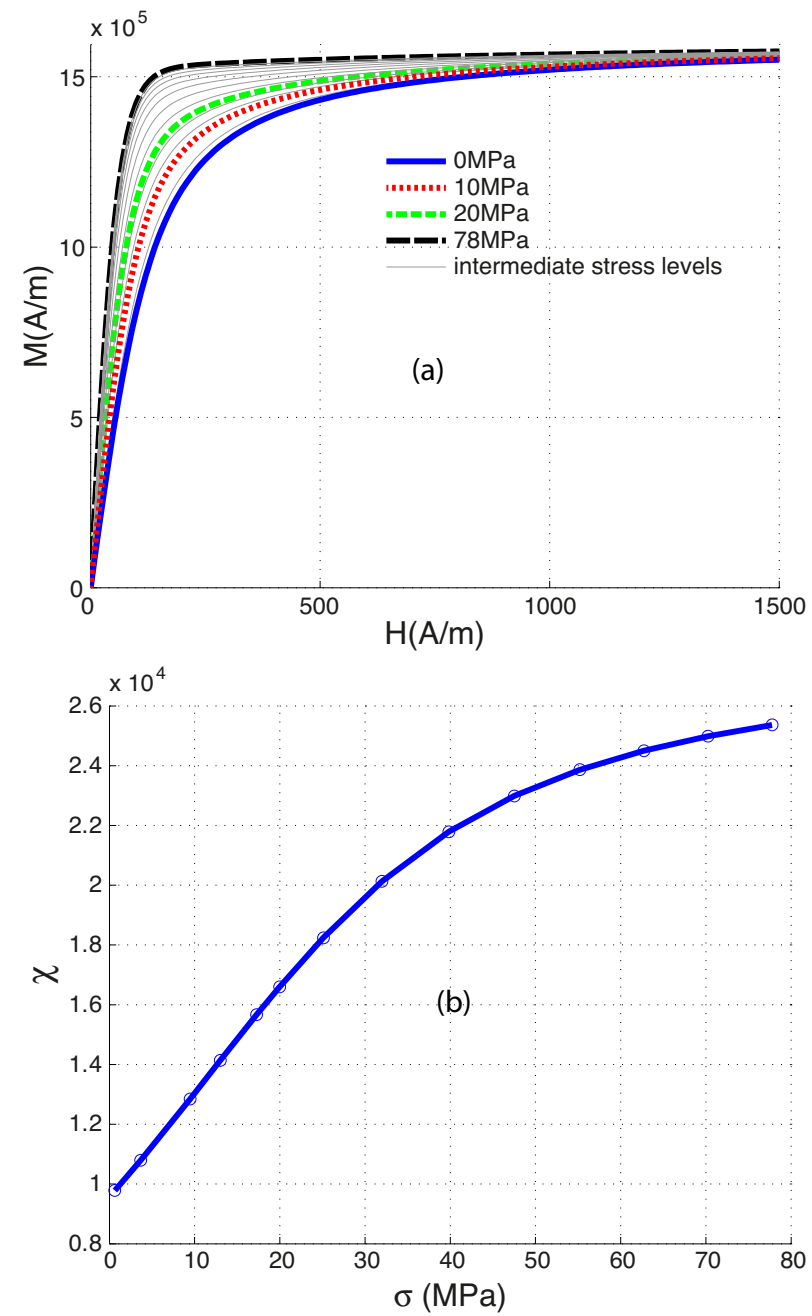

Figure 7: Effect of uniaxial stress on magnetization behavior (a) and initial susceptibility (b) - modeling results without second order stress effect - NO 3wt.\%Si-Fe. 

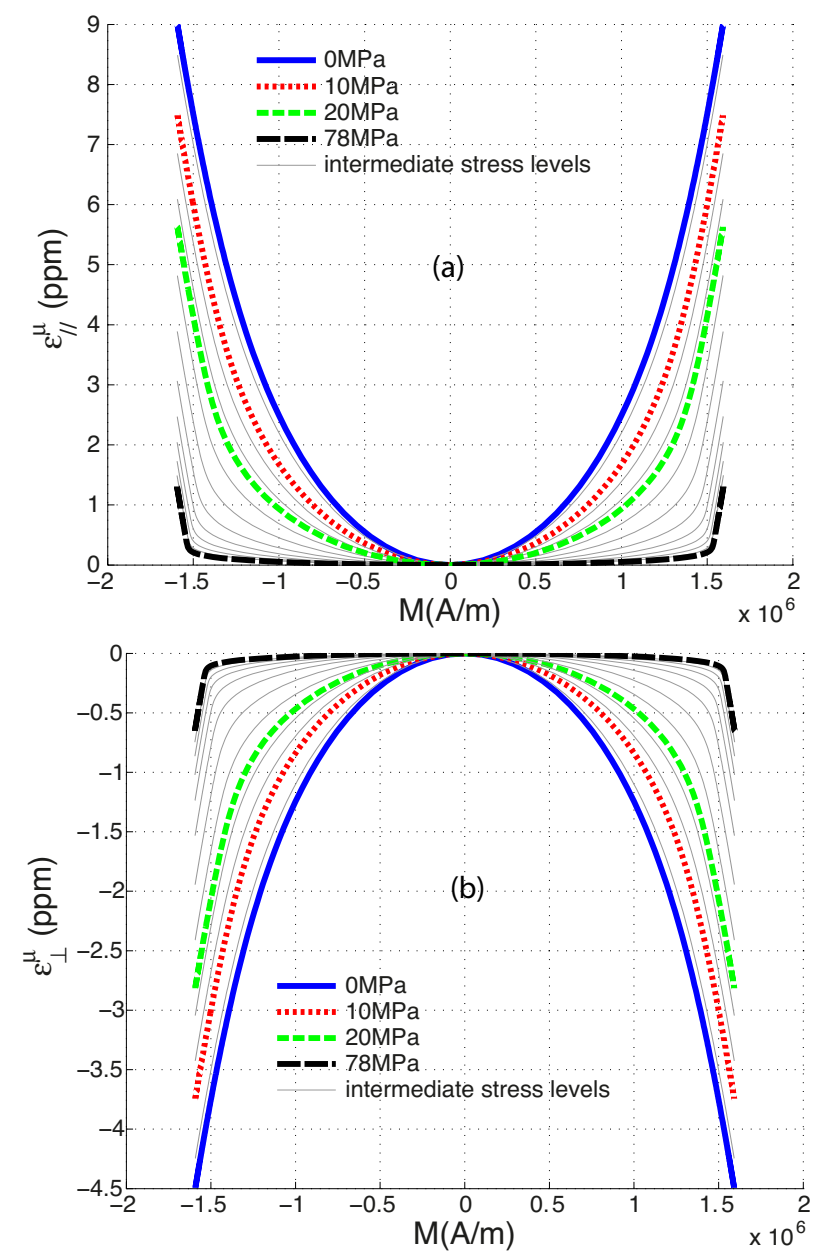

Figure 8: Effect of uniaxial stress on magnetostriction strain: longitudinal (a) and transversal (b) - modeling results without second order stress effect - NO 3wt.\%Si-Fe. 

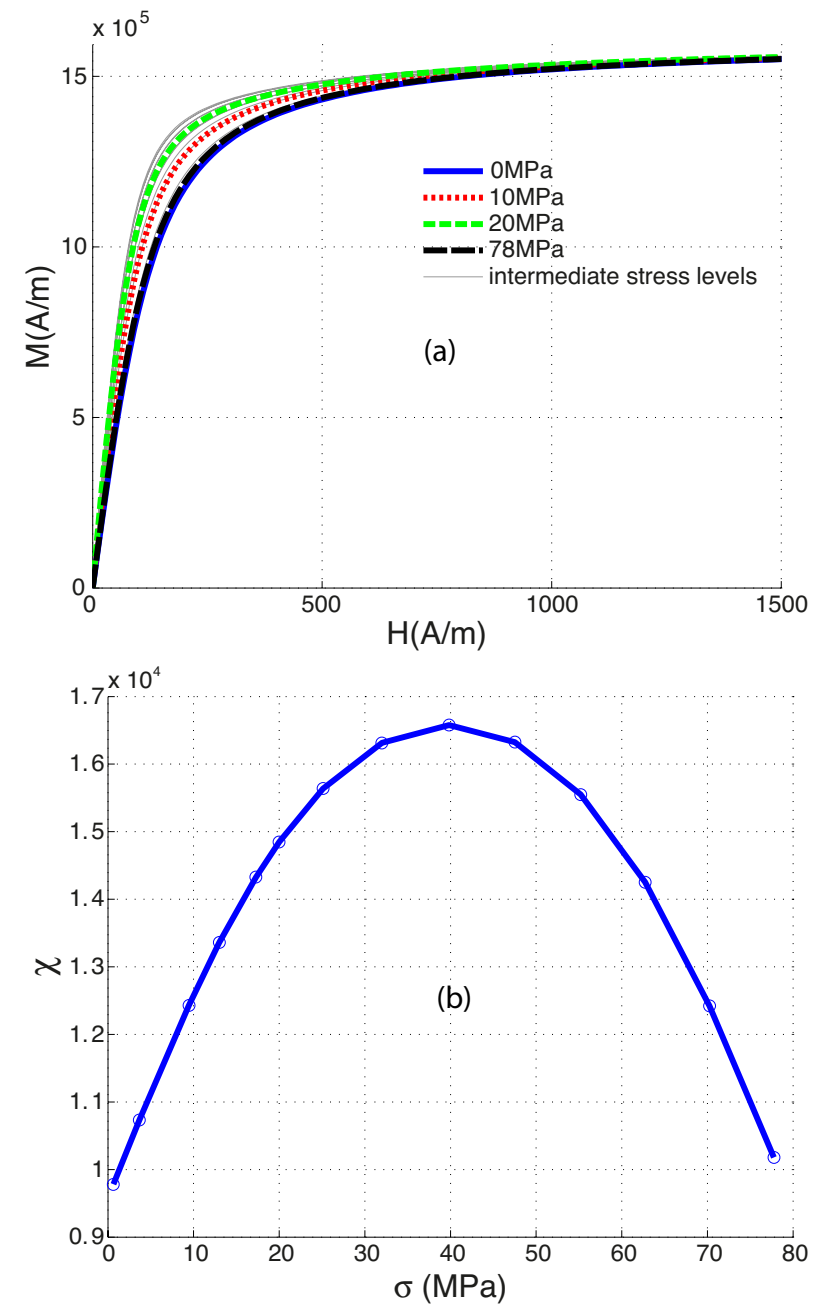

Figure 9: Effect of uniaxial stress on magnetization behavior (a) and initial susceptibility (b) - modeling results using the second order stress effect - NO 3wt.\%Si-Fe. 

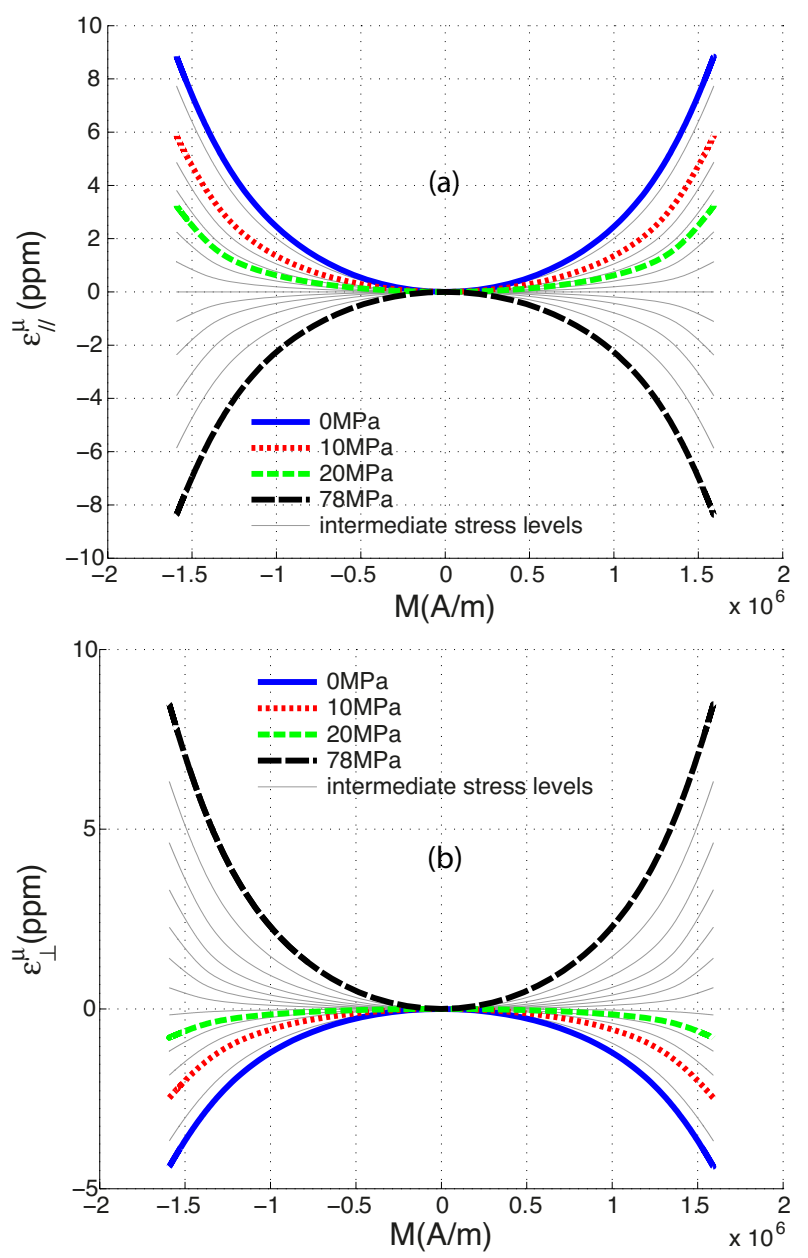

Figure 10: Effect of uniaxial stress on magnetostriction strain: longitudinal (a) and transversal (b) - modeling results using the second order stress effect - NO 3wt.\% $\mathrm{Si}-\mathrm{Fe}$. 

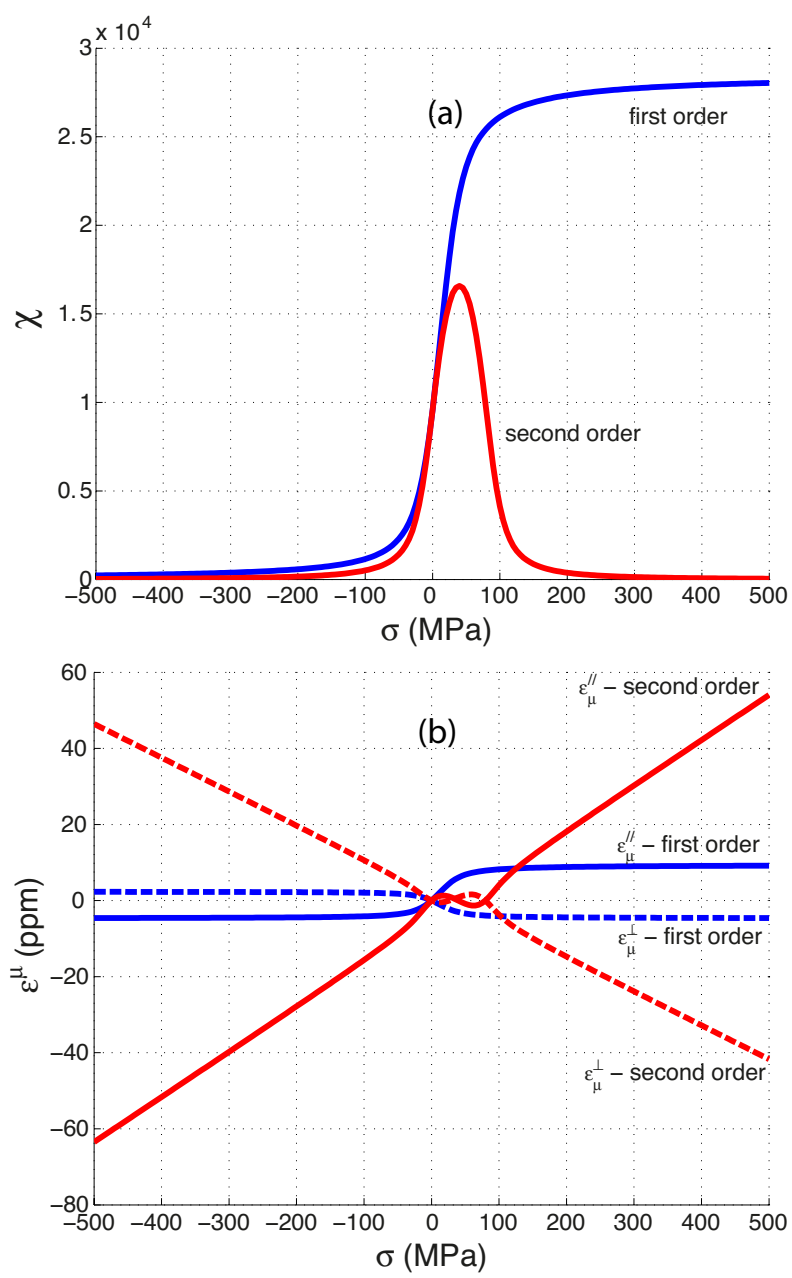

Figure 11: Effect of wide stress range on initial susceptibility (a) and longitudinal and transversal $\Delta \mathrm{E}$ effect (b) - effect of first order or second order expression using the simplified modeling - NO 3wt.\%Si-Fe. 

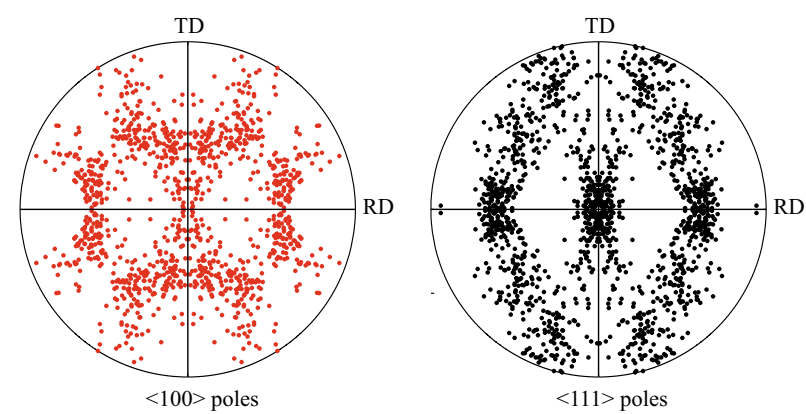

Figure 12: Discrete pole figures (396 orientations) for a NO 3wt.\%Fe-Si alloy [23]
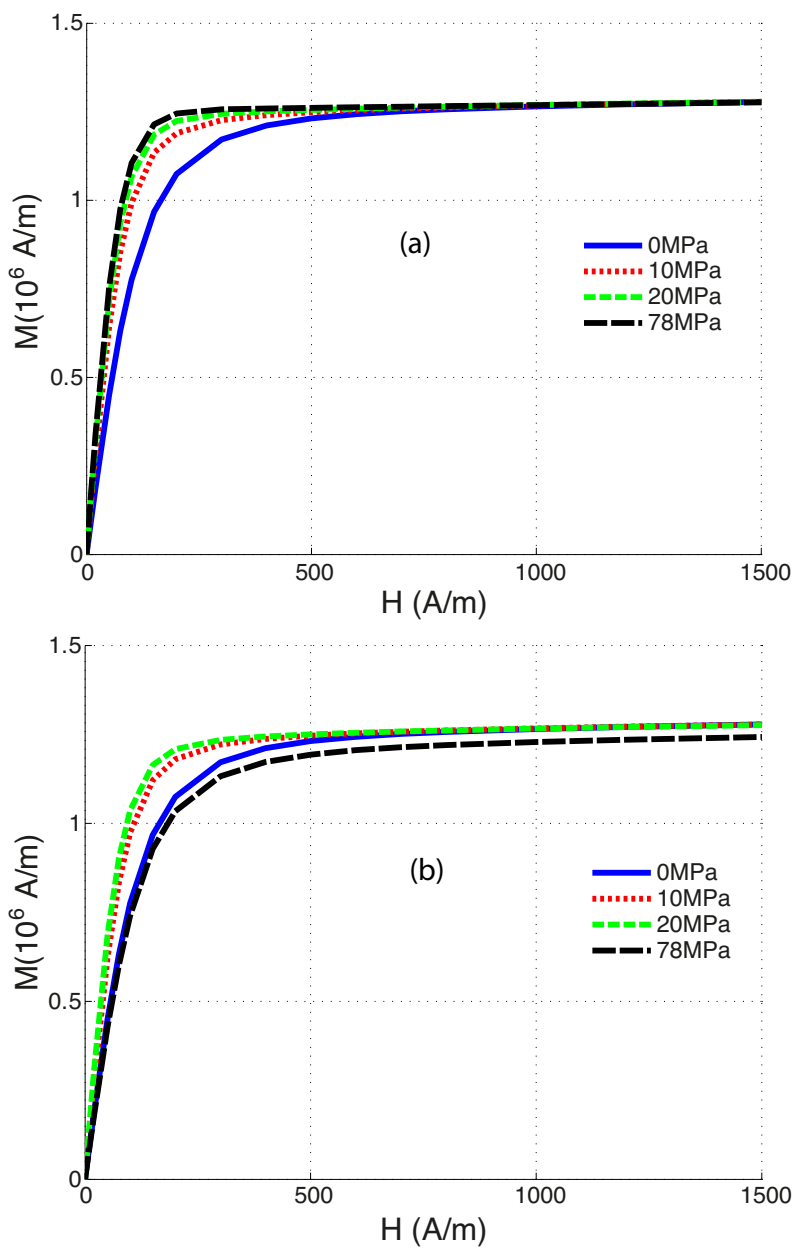

Figure 13: Multiscale modeling result of the effect of uniaxial stress on magnetization behavior: (a) first order stress effect; (b) secondorder stress effect - NO 3wt.\% Si-Fe. 


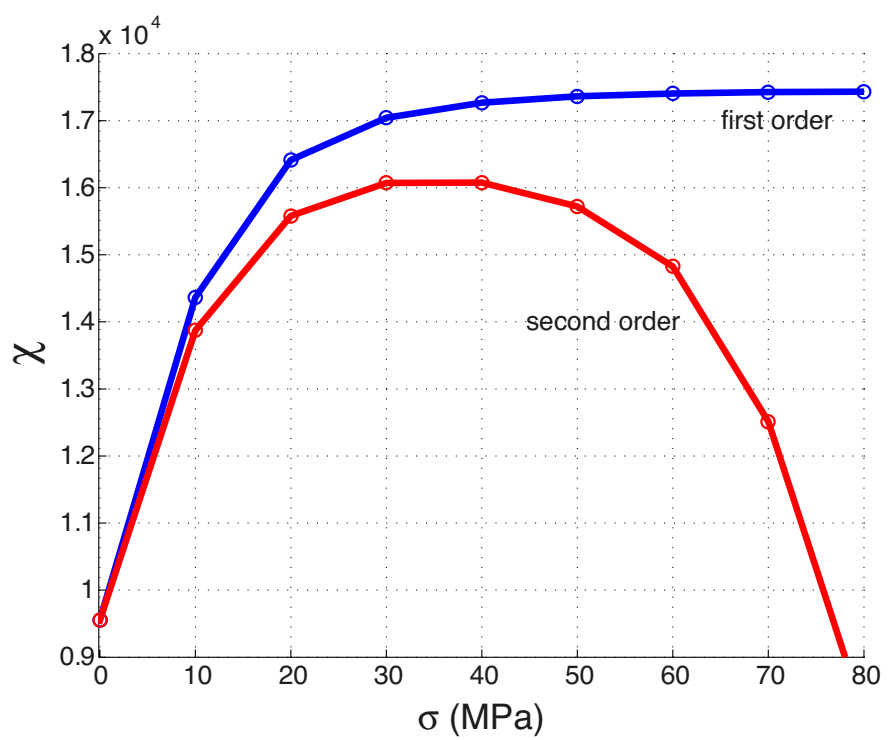

Figure 14: Multiscale modeling result of the effect of uniaxial stress on initial susceptibility: comparison between first order and second order stress effect - NO 3wt.\%Si-Fe. 

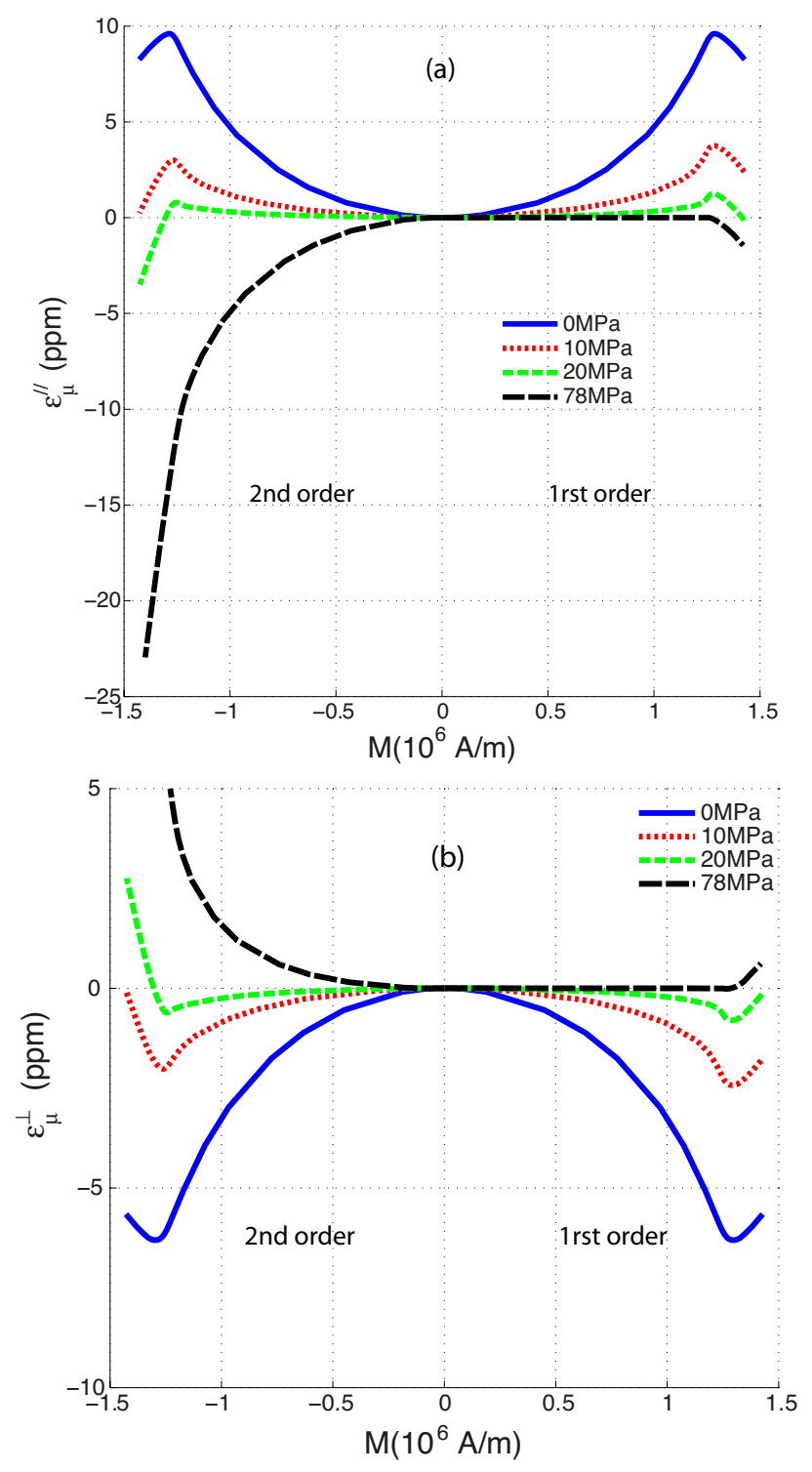

Figure 15: Multiscale modeling result of the effect of uniaxial stress on magnetostriction behavior: (a) longitudinal deformation (first and second order stress effect); (b) transversal deformation (first and second order stress effect) - NO 3wt.\%Si-Fe. 

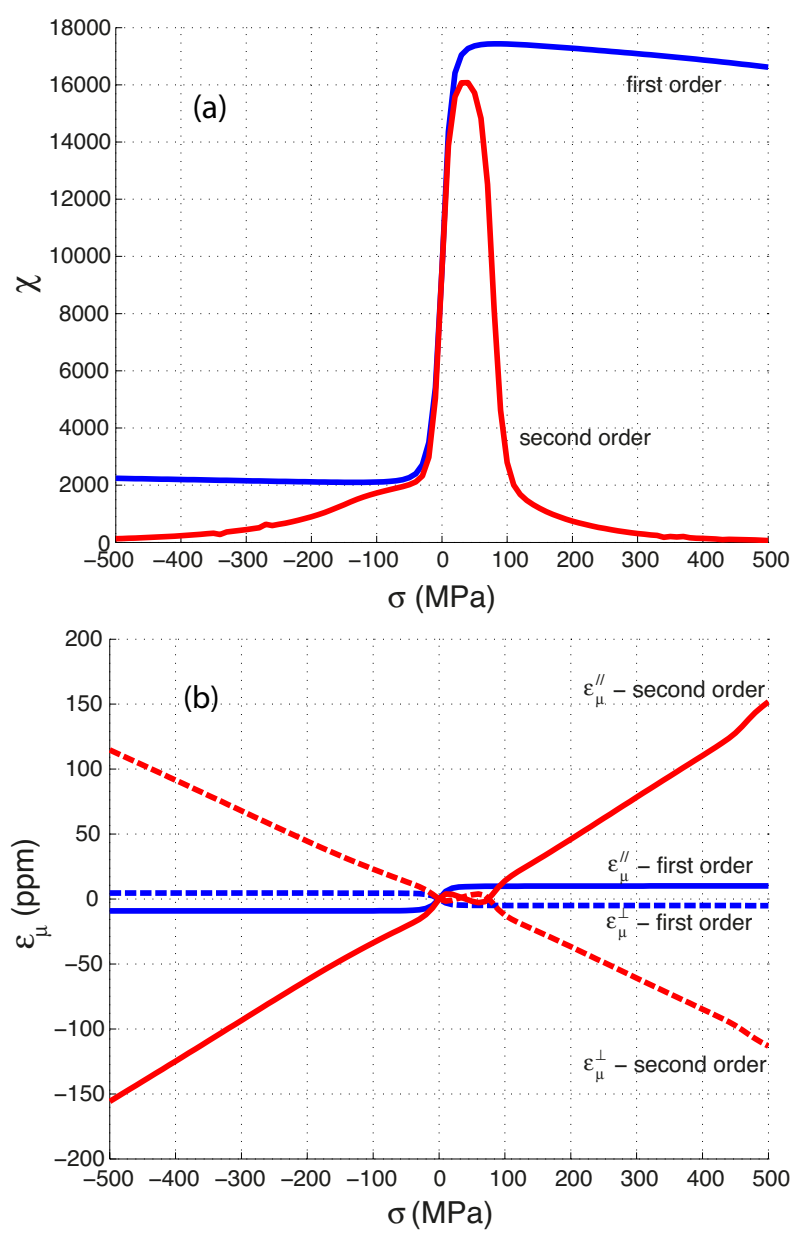

Figure 16: Multiscale modeling result of large uniaxial stress range on initial susceptibility (a) and longitudinal and transversal $\Delta \mathrm{E}$ effect (b)- effect of 1rst order or 2nd order expression NO 3wt.\%Si-Fe. 